\title{
Immunoinformatic Analysis of Calcium-Dependent Protein Kinase 7 (CDPK7) Showed Potential Targets for Toxoplasma gondii Vaccine
}

\author{
Ali Taghipour $\mathbb{D}^{1},{ }^{1}$ Sanaz Tavakoli $\mathbb{D},{ }^{2}$ Mohamad Sabaghan $\left(\mathbb{D},{ }^{3}\right.$ Masoud Foroutan $\left(\mathbb{D},{ }^{4}\right.$ \\ Hamidreza Majidiani $\left(\mathbb{D},{ }^{5}\right.$ Shahrzad Soltani $\mathbb{D}^{4}{ }^{4}$ Milad Badri ${ }^{(D)},{ }^{6}$ Ali Dalir Ghaffari $\mathbb{D}^{1}{ }^{1}$ \\ and Sheyda Soltani $\mathbb{D}^{4}$
}

\author{
${ }^{1}$ Department of Parasitology, Faculty of Medical Sciences, Tarbiat Modares University, Tehran, Iran \\ ${ }^{2}$ Department of Parasitology and Mycology, School of Medicine, Isfahan University of Medical Sciences, Isfahan, Iran \\ ${ }^{3}$ Behbahan Faculty of Medical Sciences, Behbahan, Iran \\ ${ }^{4}$ USERN Office, Abadan University of Medical Sciences, Abadan, Iran \\ ${ }^{5}$ Zoonotic Diseases Research Center, Ilam University of Medical Sciences, Ilam, Iran \\ ${ }^{6}$ Medical Microbiology Research Center, Qazvin University of Medical Sciences, Qazvin, Iran
}

Correspondence should be addressed to Mohamad Sabaghan; sabaghan.m@ajums.ac.ir

and Masoud Foroutan; masoud_foroutan_rad@yahoo.com

Received 10 April 2021; Revised 10 June 2021; Accepted 22 June 2021; Published 12 July 2021

Academic Editor: José F. Silveira

Copyright (C) 2021 Ali Taghipour et al. This is an open access article distributed under the Creative Commons Attribution License, which permits unrestricted use, distribution, and reproduction in any medium, provided the original work is properly cited.

Apicomplexan parasites, including Toxoplasma gondii (T. gondii), express different types of calcium-dependent protein kinases (CDPKs), which perform a variety of functions, including attacking and exiting the host cells. In the current bioinformatics study, we have used several web servers to predict the basic features and specifications of the CDPK7 protein. The findings showed that CDPK7 protein has 2133 amino acid residues with an average molecular weight (MW) of 219085.79 D. The aliphatic index with 68.78 and grand average of hydropathicity (GRAVY) with -0.331 score were estimated. The outcomes of current research showed that the CDPK7 protein included 502 alpha-helix, 1311 random coils, and 320 extended strands with GOR4 method. Considering the Ramachandran plot, the favored region contains more than $92 \%$ of the amino acid residues. In addition, evaluation of antigenicity and allergenicity showed that CDPK7 protein has immunogenic and nonallergenic nature. The present research provides key data for more animal-model study on the CDPK7 protein to design an efficient vaccine against toxoplasmosis in the future.

\section{Introduction}

Toxoplasma gondii is a prevalent intracellular protozoan, which can infect a broad spectrum of mammals (i.e., human) and birds $[1,2]$. Oocysts are the potential infective form in the life cycle of the parasite. Feline species as the only definitive hosts can contaminate the environment by shedding unsporulated oocysts through feces [3]. T. gondii is transferred by water/vegetables contaminated via mature oocysts and consumption of raw or semicooked meat from infected animals, vertical transmission from infected preg- nant mothers to neonates, and blood transfusion [4-7]. Approximately one-third of human society has been exposed to $T$. gondii, worldwide $[5,8,9]$. Often $T$. gondii infection among immunocompetent people is asymptomatic or demonstrates mild symptoms, whereas in immunocompromised patients, it can cause a various range of clinical symptoms $[6,9,10]$. Toxoplasmosis in immunocompromised subjects can cause repeated attacks in the brain and manifests as encephalitis [11]. Moreover, toxoplasmosis in pregnant women can cause blindness, microcephaly, and mental retardation in the infant $[6,12]$. Different factors, such as host's 
Journal of Parasitology Research

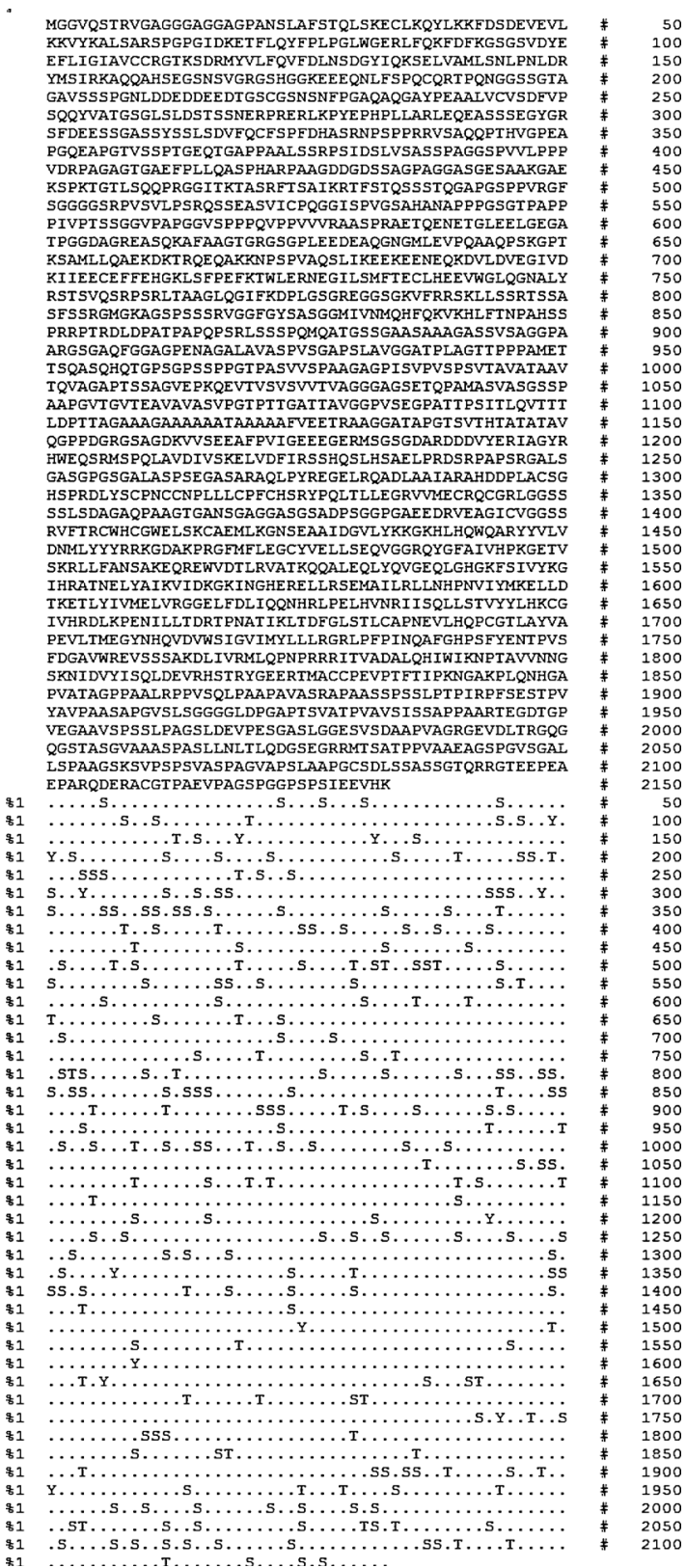

(a)

Figure 1: Continued. 


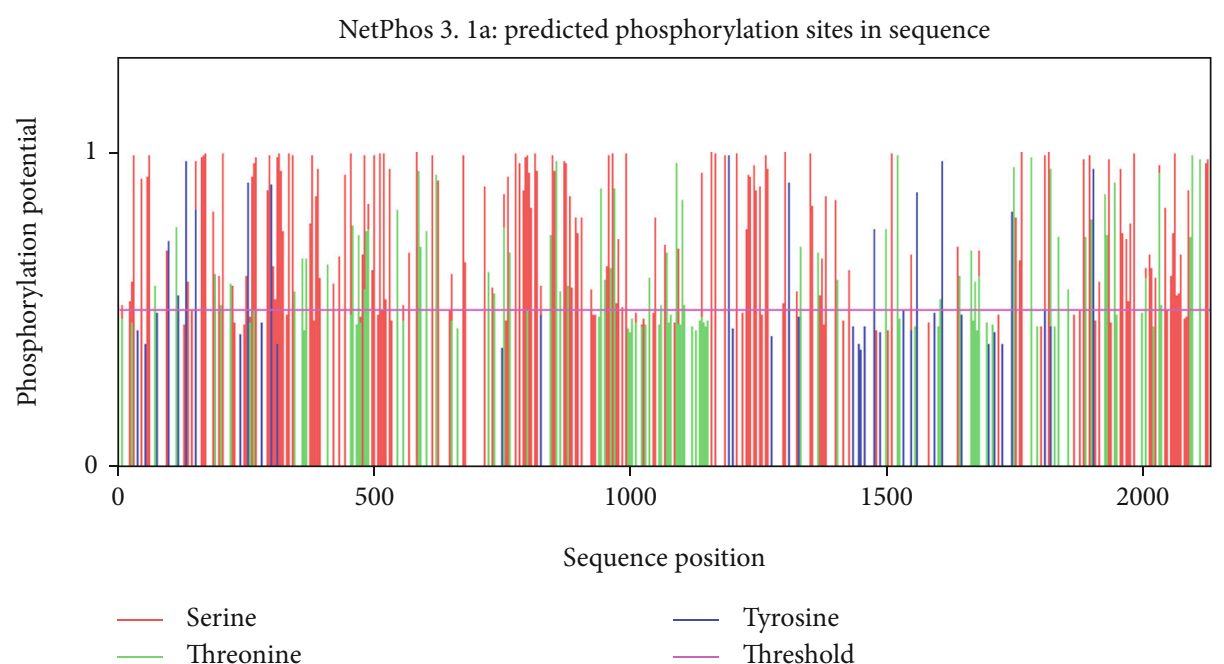

(b)

FIGURE 1: NetPhos server output for CDPK7 phosphorylation sites. (a) The number of predicted sites, based on S (serine), $\mathrm{T}$ (threonine), and $\mathrm{Y}$ (tyrosine); (b) prediction diagram of CDPK7 phosphorylation sites.

TABLE 1: The acylation sites of CDPK7 sequence.

\begin{tabular}{|c|c|c|c|}
\hline ID & Position & Peptide & Score \\
\hline TGME49_228750 CDPK7 (T. gondii) & 34 & STQLSKECLKQYLKK & 1.129 \\
\hline TGME49_228750 CDPK7 (T. gondii) & 109 & FLIGIAVCCRGTKSD & 1.996 \\
\hline TGME49_228750 CDPK7 (T. gondii) & 110 & LIGIAVCCRGTKSDR & 5.494 \\
\hline TGME49_228750 CDPK7 (T. gondii) & 187 & QNLFSPQCQRTPQNG & 0.526 \\
\hline TGME49_228750 CDPK7 (T. gondii) & 222 & DEEDTGSCGSNSNFP & 5.293 \\
\hline TGME49_228750 CDPK7 (T. gondii) & 244 & YPEAALVCVSDFVPS & 3.693 \\
\hline TGME49_228750 CDPK7 (T. gondii) & 321 & SLSDVFQCFSPFDHA & 0.984 \\
\hline TGME49_228750 CDPK7 (T. gondii) & 524 & SSEASVICPQGGISP & 2.536 \\
\hline TGME49_228750 CDPK7 (T. gondii) & 706 & VDKIIEECEFFEHGK & 0.403 \\
\hline TGME49_228750 CDPK7 (T. gondii) & 736 & ILSMFTECLHEEVWG & 1.821 \\
\hline TGME49_228750 CDPK7 (T. gondii) & 1298 & AHDDPLACSGHSPRD & 5.591 \\
\hline TGME49_228750 CDPK7 (T. gondii) & 1309 & SPRDLYSCPNCCNPL & 1.744 \\
\hline TGME49_228750 CDPK7 (T. gondii) & 1312 & DLYSCPNCCNPLLLC & 8.015 \\
\hline TGME49_228750 CDPK7 (T. gondii) & 1313 & LYSCPNCCNPLLLCP & 7.05 \\
\hline TGME49_228750 CDPK7 (T. gondii) & 1319 & CCNPLLLCPFCHSRY & 2.719 \\
\hline TGME49_228750 CDPK7 (T. gondii) & 1322 & PLLLCPFCHSRYPQL & 2.865 \\
\hline TGME49_228750 CDPK7 (T. gondii) & 1340 & EGRVVMECRQCGRLG & 2.295 \\
\hline TGME49_228750 CDPK7 (T. gondii) & 1343 & VVMECRQCGRLGGSS & 2.929 \\
\hline TGME49_228750 CDPK7 (T. gondii) & 1395 & DRVEAGICVGGSSRV & 5.406 \\
\hline TGME49_228750 CDPK7 (T. gondii) & 1406 & SSRVFTRCWHCGWEL & 0.108 \\
\hline TGME49_228750 CDPK7 (T. gondii) & 1409 & VFTRCWHCGWELSKC & 1.424 \\
\hline TGME49_228750 CDPK7 (T. gondii) & 1416 & CGWELSKCAEMLKGN & 4.272 \\
\hline TGME49_228750 CDPK7 (T. gondii) & 1474 & GFMFLEGCYVELLSE & 1.626 \\
\hline TGME49_228750 CDPK7 (T. gondii) & 1649 & TVYYLHKCGIVHRDL & 1.164 \\
\hline TGME49_228750 CDPK7 (T. gondii) & 1683 & DFGLSTLCAPNEVLH & 1.6 \\
\hline TGME49_228750 CDPK7 (T. gondii) & 1693 & NEVLHQPCGTLAYVA & 1.927 \\
\hline TGME49_228750 CDPK7 (T. gondii) & 1828 & GEERTMACCPEVPTF & 4.139 \\
\hline TGME49_228750 CDPK7 (T. gondii) & 1829 & EERTMACCPEVPTFT & 7.362 \\
\hline TGME49_228750 CDPK7 (T. gondii) & 2080 & PSLAAPGCSDLSSAS & 3.862 \\
\hline TGME49_228750 CDPK7 (T. gondii) & 2110 & ARQDERACGTPAEVP & 6.173 \\
\hline
\end{tabular}




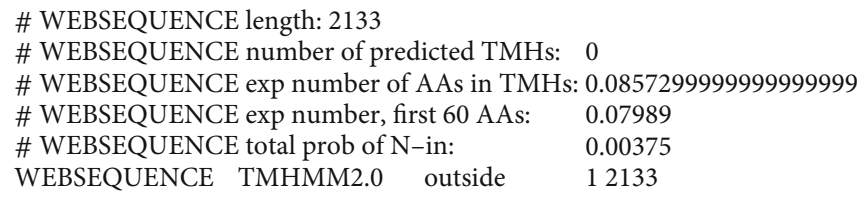

(a)

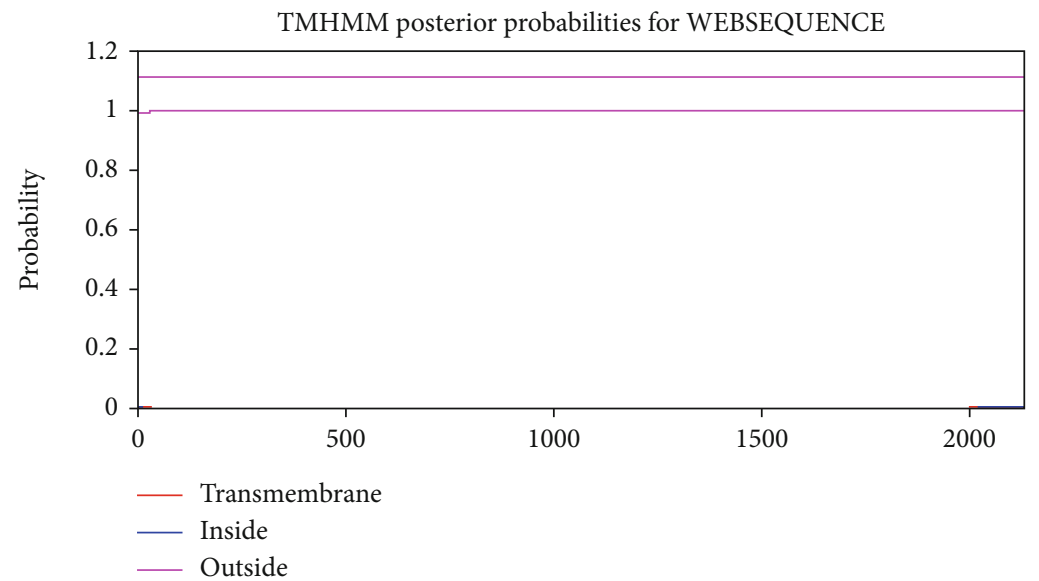

(b)

FIGURE 2: Transmembrane domains expected in CDPK7 protein. (a) Some statistics and a list of the location of the predicted transmembrane helices and the predicted location of the intervening loop regions. Length: the length of the protein sequence; number of predicted TMHs: the number of predicted transmembrane helices; Exp number of AAs in TMHs: the expected number of amino acids in transmembrane helices. If this number is larger than 18, it is very likely to be a transmembrane protein (or have a signal peptide); Exp number, first 60 AAs: the expected number of amino acids in transmembrane helices in the first 60 amino acids of the protein. If this number is more than a few, you should be warned that a predicted transmembrane helix in the $\mathrm{N}$-term could be a signal peptide; total prob of $\mathrm{N}$-in: the total probability that the $\mathrm{N}$-term is on the cytoplasmic side of the membrane; (b) transmembrane domains expected in CDPK7 protein. (b) Analysis of the transmembrane domains of CDPK7.

immune system status, genetic background, age, gender, contact with infected cats, environmental conditions, and diet and cultural habits, as well as the protozoan genotype, can affect the morbidity and mortality rate of Toxoplasma infection $[13,14]$.

Today, treatment of toxoplasmosis with conventional drugs can just limit the proliferation of tachyzoites at the beginning of infection, while these drugs cannot eradicate cystic forms of parasites in host tissue $[15,16]$. In addition, taking these medications in pregnant women can have serious side effects, such as the possibility of teratogenic effects on the fetus [17]. Hence, the discovery and design of an effective vaccine to control and prevent toxoplasmosis is very important, especially in humans and domestic animals. In this regard, various in silico-based studies suggest various antigens as suitable candidates for vaccine design [18-32]. Calcium-dependent protein kinases (CDPKs) are a class of serine/threonine kinases that express in apicomplexans, ciliates, and plants [33]. In T. gondii as a member of the apicomplexan parasites, several CDPKs have been identified involving in critical functions in the different stages of the life cycle of parasite, including gliding motility (surface translocation), entry into (invasion), and exit from (egress) of host cells [34]. The CDPK7 is a crucial enzyme for division, growth, and maintenance of structural integrity of the Toxoplasma centrosome. As a result, TgCDPK7 knockdown is suggested as an important goal in achieving the right vaccine [35].
Computer-aided evaluation of different T. gondii proteins involved in various stages of life cycle can open new doors towards recognizing potent vaccine candidates through identification of highly immunogenic, nonallergenic, and nontoxic B- and T-cell epitopes [36]. Thereby, the present in silico study was performed to evaluate the crucial biochemical features and immunogenic epitopes of the CDPK7 protein by means of different bioinformatics servers.

\section{Methods}

2.1. CDPK7 Sequence. For this purpose, ToxoDB online website was used to obtain the whole amino acid sequence of $T$. gondii CDPK7 protein.

2.2. Physicochemical Characterization. We used the Expasy ProtParam online server to predict the physicochemical parameters of CDPK7 [37].

2.3. Prediction of Posttranslational Modification (PTM) Sites. The NetPhos 3.1 online tool was applied to predict phosphorylation location, and the CSS-Palm online server was applied to predict acylation location of the CDPK7 [38, 39].

2.4. Transmembrane Domains and Subcellular Location. The transmembrane regions and subcellular localization of $T$. gondii CDPK7 protein were assessed utilizing the TMHMM 2.0 and PSORT II web servers, respectively [38]. 


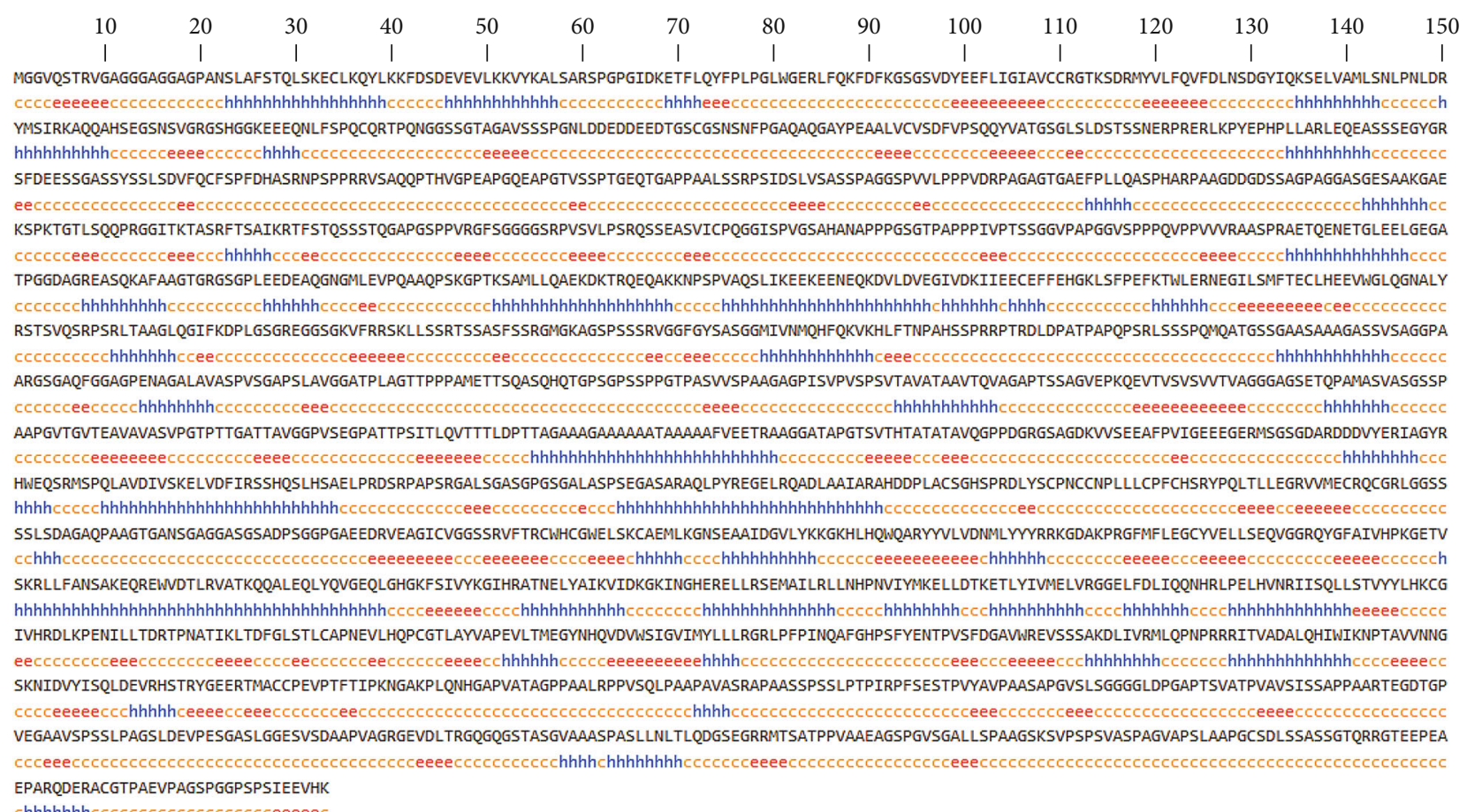

(a)

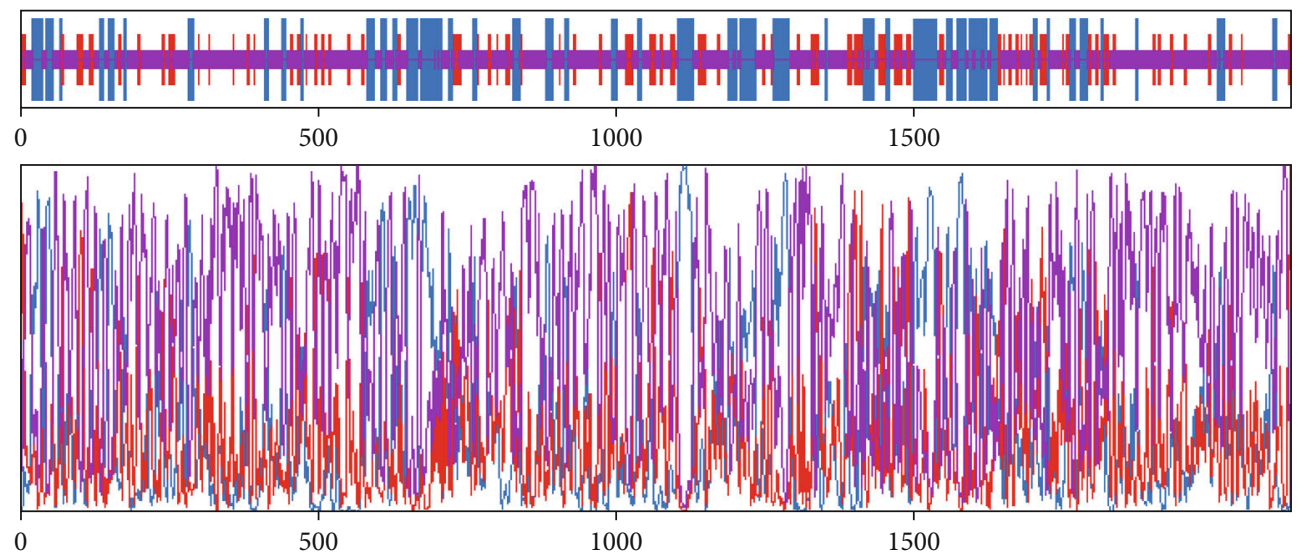

(b)

FIgURE 3: (a) GOR4 server results suggested that CDPK7 encompasses 502 alpha-helix, 320 extended strands, and 1311 random coils in secondary structure; (b) graphical result of the secondary structure prediction of CDPK7 using the GOR4 online server.

2.5. Secondary and Tertiary Structures. In this study, we employed the Garnier-Osguthorpe-Robson 4 (GOR4) online tool to forecast the secondary structure of CDPK7 protein [40]. Consequently, the three-dimensional (3D) model structures was used by SWISS-MODEL [38, 41].

2.6. The 3D Modeled Structure Refinement and Validation. GalaxyRefine was selected to develop and refine the quality of the template-based protein prediction [42]. To the Ramachandran plot validated the 3D structure of the protein, the SWISS-MODEL software was applied [43]. ProSA-web was used for evaluation of the whole quality of the model [44].

2.7. Linear and Conformational B-Cell Epitopes. We used a web-based Bcepred server to predict continuous B-cell epi- topes exploiting physicochemical characteristics [45]. An online server of ABCpred was applied to predict B-cell epitopes [46]. Using the immune epitope database (IEDB), hydrophilicity [47], Bepipred linear epitope prediction [48], antigenicity [49], surface accessibility [50], beta-turn [51], and flexibility [52] were predicted. Afterwards, discontinuous B-cell epitopes were appraised by ElliPro [53] from the 3D structure of protein epitopes.

2.8. MHC-I and MHC-II Epitopes. To this aim, we used the IEDB website to evaluate the half-maximal inhibitory concentration $\left(\mathrm{IC}_{50}\right)$ values of peptides that bind to the main histocompatibility complex (MHC) class I and class II molecules for CDPK7 $[54,55]$. All predicted epitopes were then 


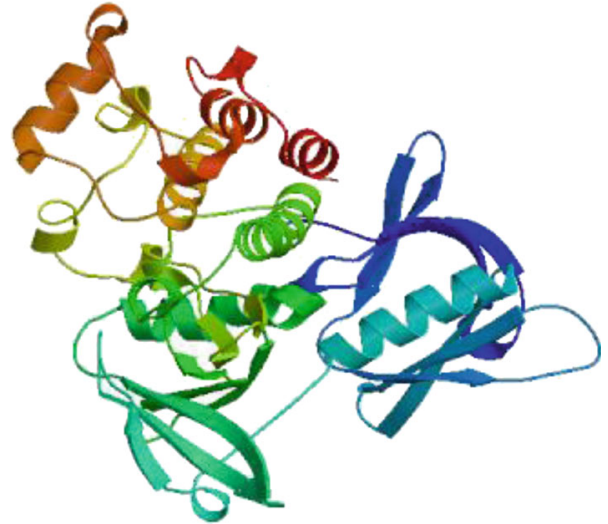

(a)

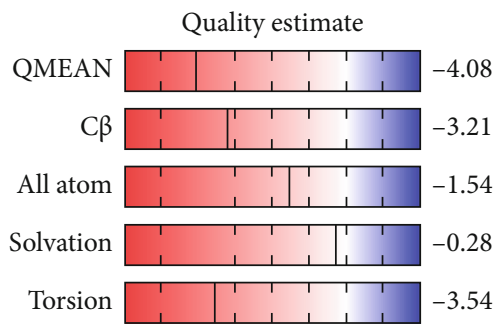

(b)

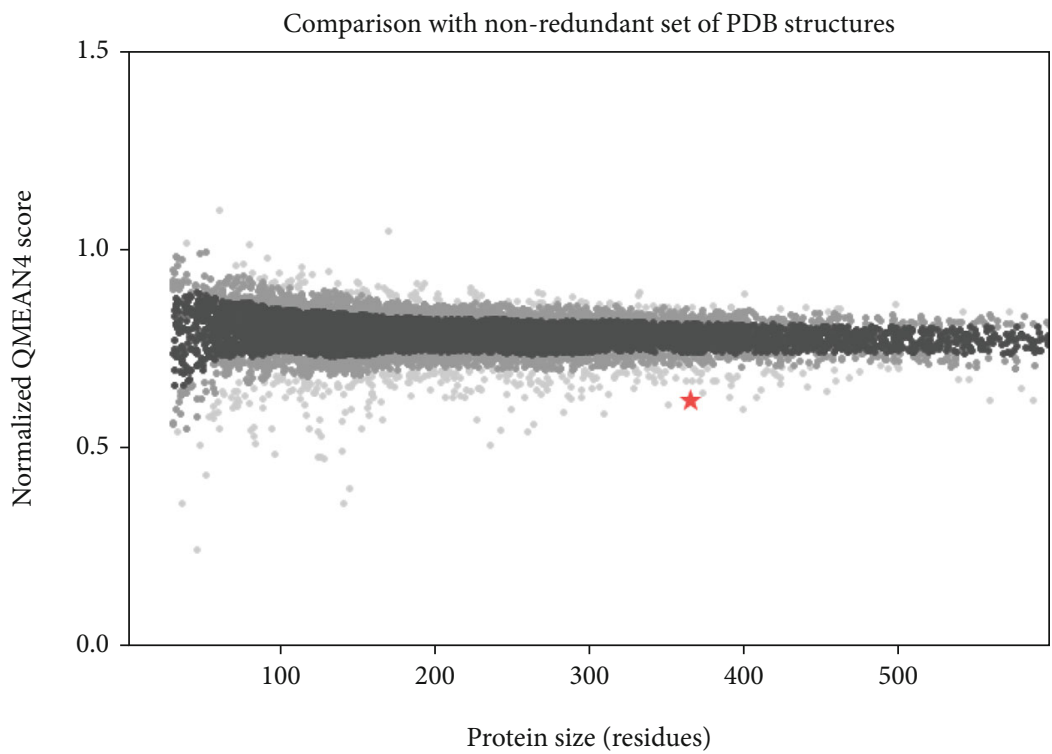

- $\mid Z$-score $\mid>2$

- $1<\mid Z$-score $\mid<2$

- $\mid Z$-score $\mid<1$

$\star \quad$ Model

(c)

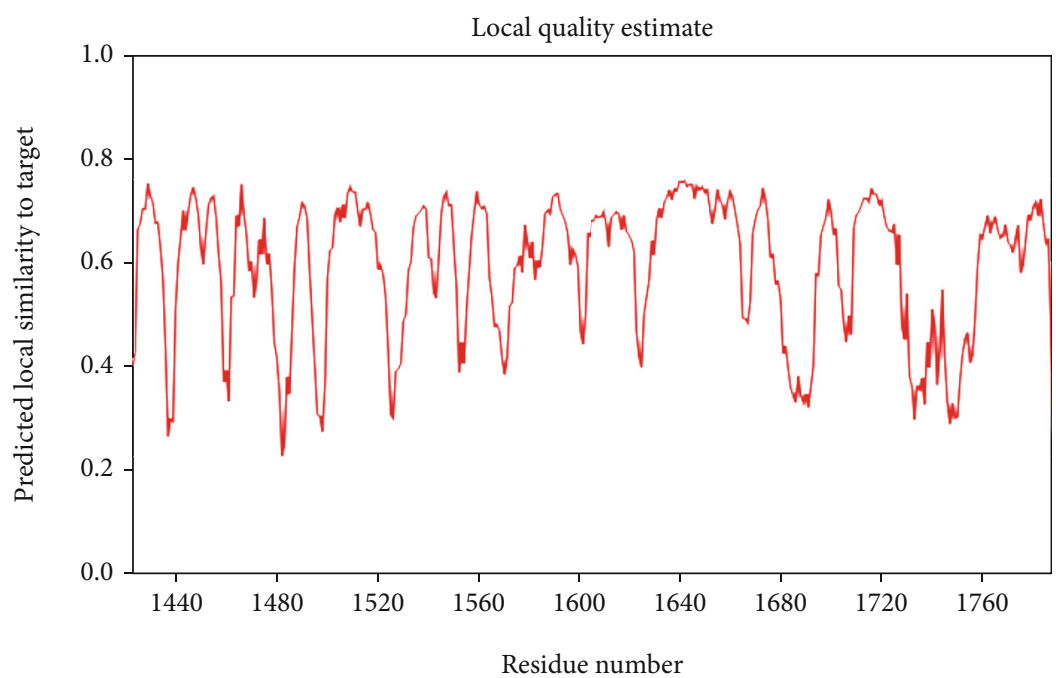

(d)

FIGURE 4: SWISS-MODEL server output. (a) Computed 3D model; (b) global quality estimate; (c) comparison with nonredundant set of PDB structures; (d) local quality estimate. 

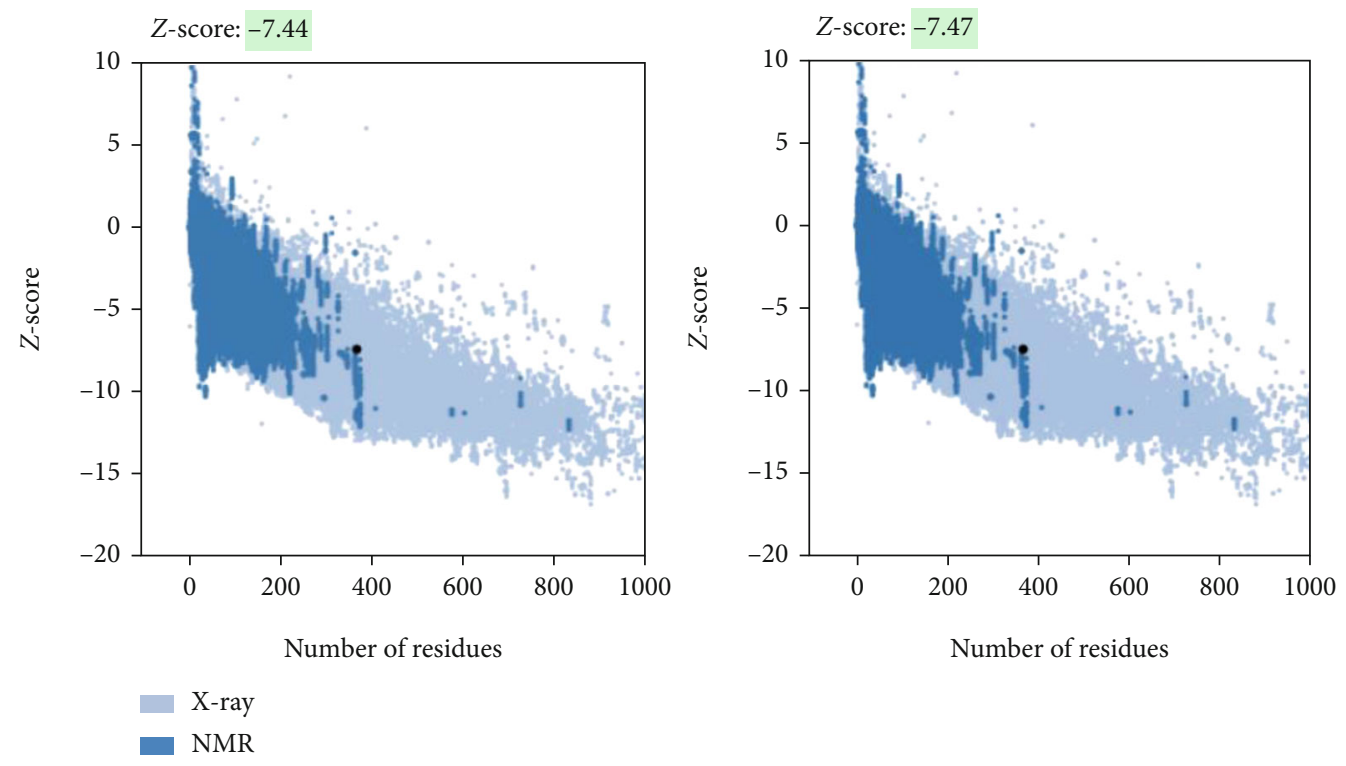

(a)
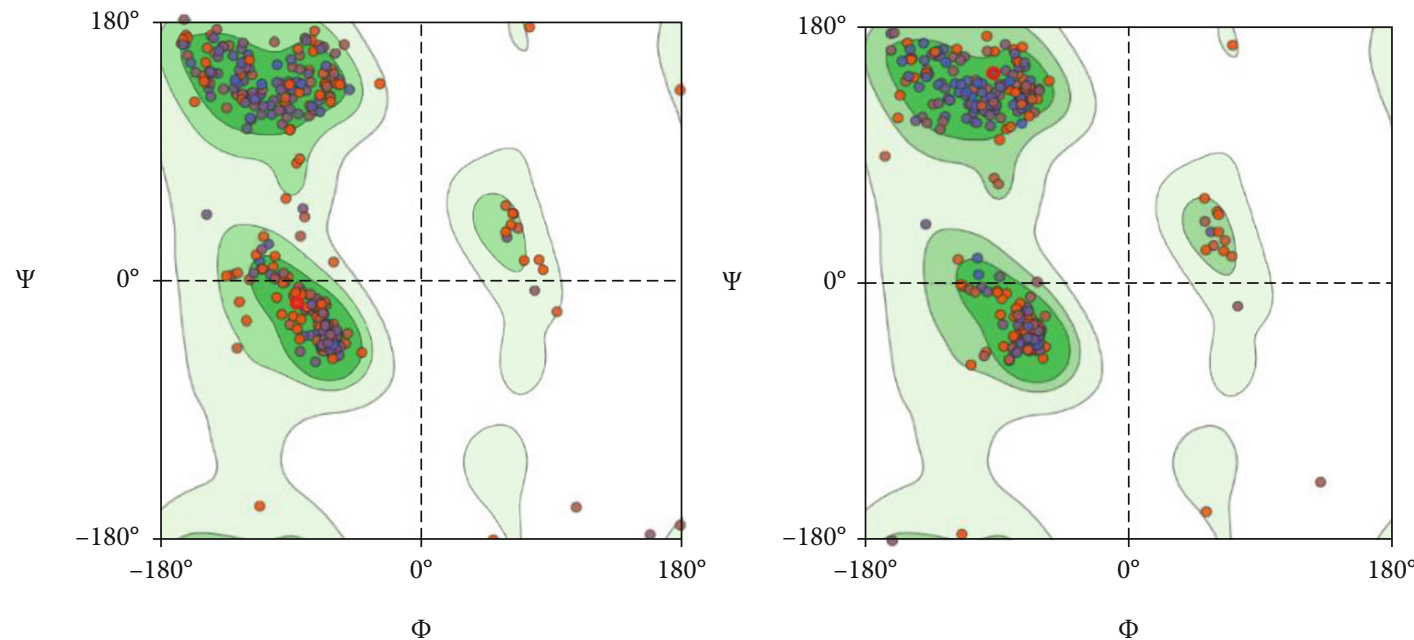

(b)

FIGURE 5: Validation of 3D model of CDPK7 protein. (a) The $Z$-score plot for 3D structure of predicted protein before and after refinement with ProSA-web server, respectively; (b) Ramachandran plot analysis of predicted structure.

evaluated in terms of antigenicity using the VaxiJen v2.0 server.

2.9. Cytotoxic T-Lymphocyte (CTL) Epitopes. We applied CTLpred online website according to $75.8 \%$ accuracy [56]. Next, all predicted epitopes were evaluated regarding antigenicity using the VaxiJen v2.0 server.

2.10. Antigenic and Allergenic Profiles. The antigenicity of the full CDPK7 sequence was estimated by VaxiJen v2.0 [57]. The allergenic profile of CDPK7 was predicted by the AllergenFP v1.0 and AllerTOP v2.0 servers $[58,59]$.

\section{Results}

3.1. General Information of CDPK7. The amino acid structure of CDPK7 was obtained from the ToxoDB server with accession no. TGME49_228750. Based on the ProtParam database, the CDPK7 protein entails of 2133 amino acid residues with molecular weight of $219085.79 \mathrm{D}$, whereas theoretical pI was 5.79. The overall number of negatively (Asp + Glu) charged residues was 209, and positively (Arg+Lys) charged residues was 178. There are a total number of 30441 atoms. The half-life of the CDPK7 was predictable at 30 hours, $>20$ hours, and $>10$ hours for mammalian (in vitro), yeast (in vivo), and Escherichia coli (in vivo), respectively. In addition, the instability index of the CDPK7 protein presented an unstable nature with a value of 53.28. In addition, the aliphatic index was calculated 68.78, and GRAVY of the protein was estimated -0.331 .

3.2. PTM Sites of CDPK7 Protein. In the present research, the results exhibited that 269 phosphorylation sites (Thr: 64, Tyr: 13, and Ser: 192) (Figures 1(a) and 1(b)) and 30 acylation 


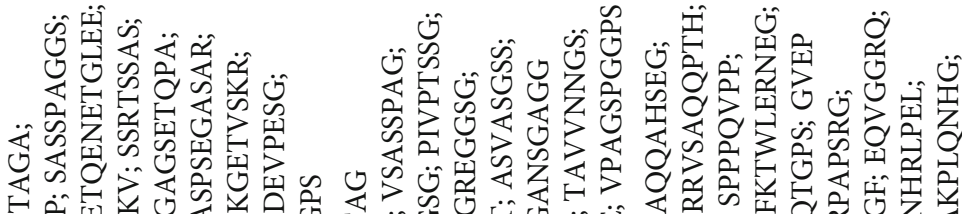

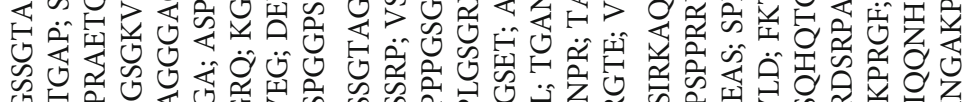
ड़

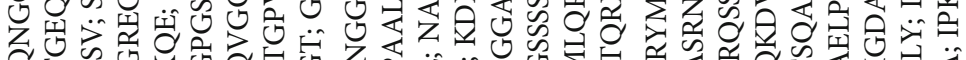

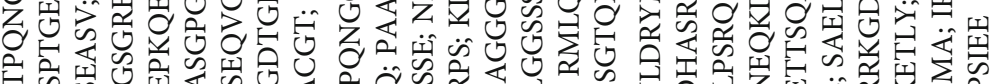

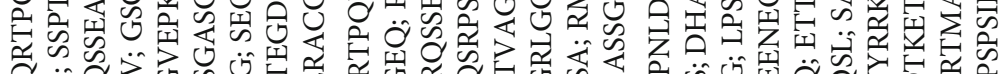

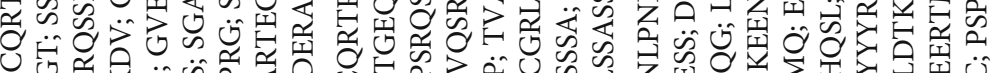
O के $\begin{aligned} & 1 \\ & \text { की }\end{aligned}$

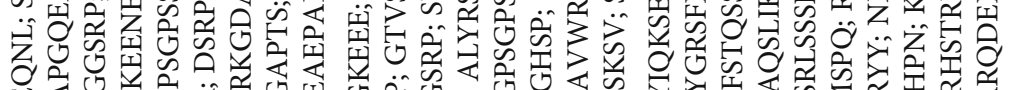

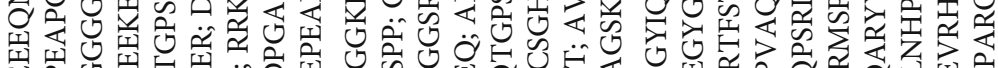

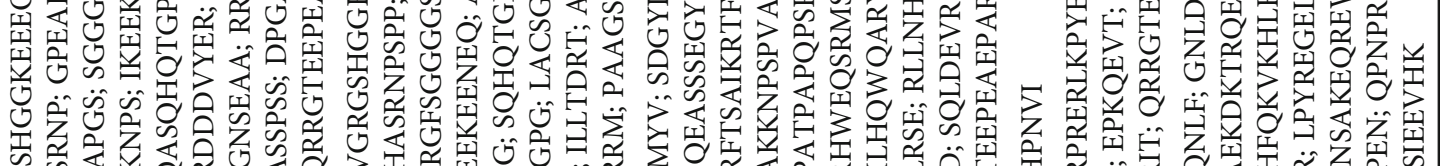

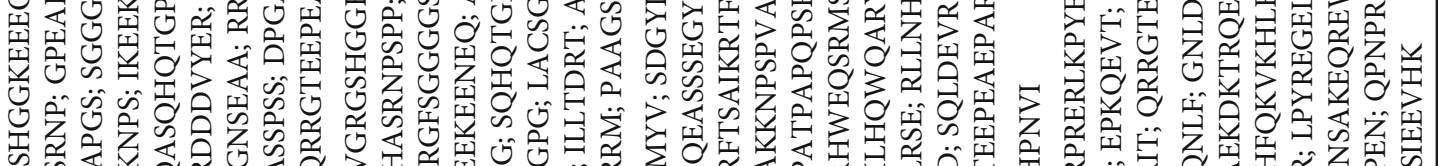

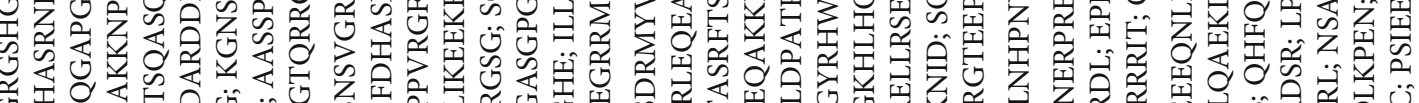

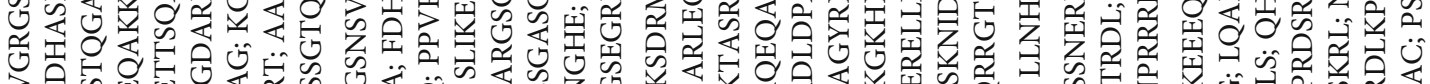

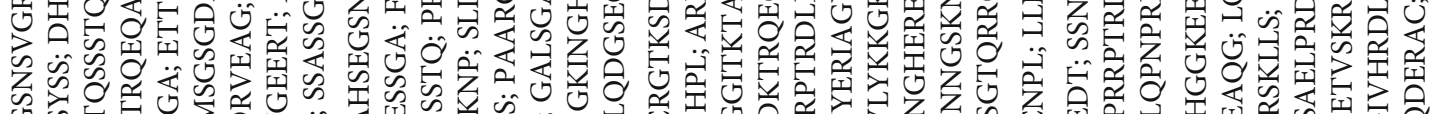

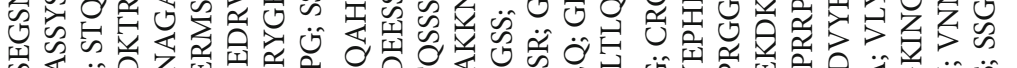

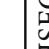

$\vec{\Phi}$

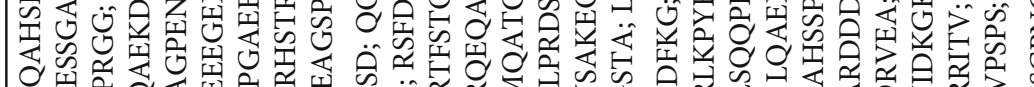

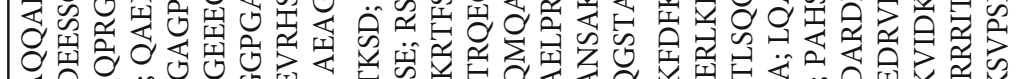

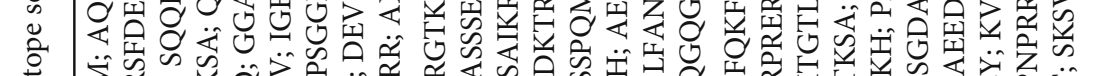

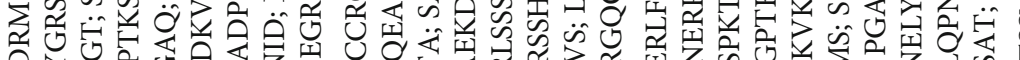

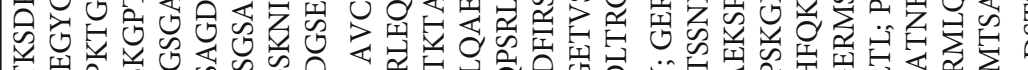

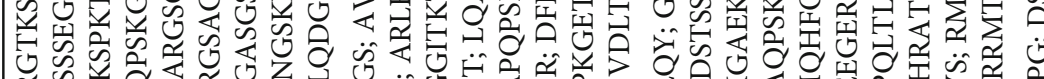

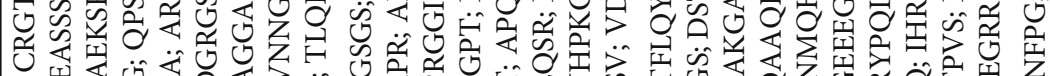

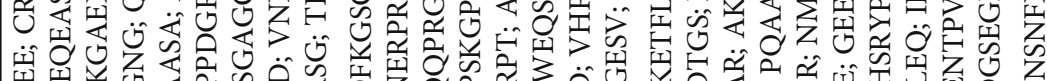

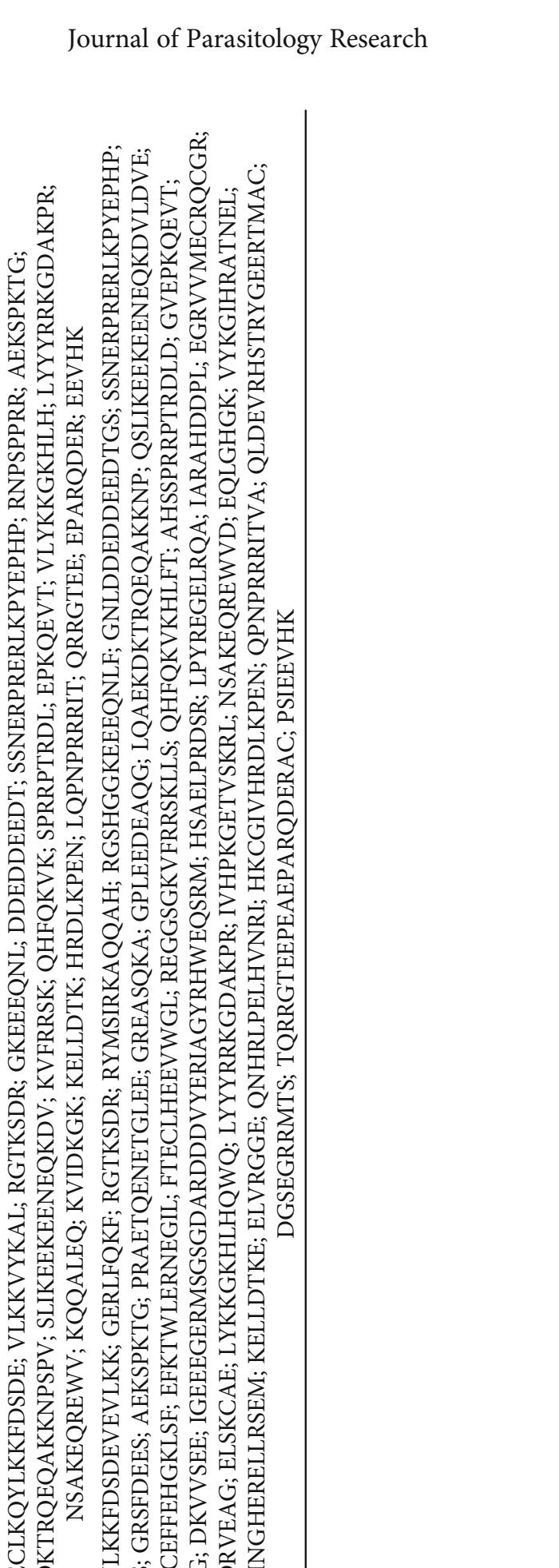

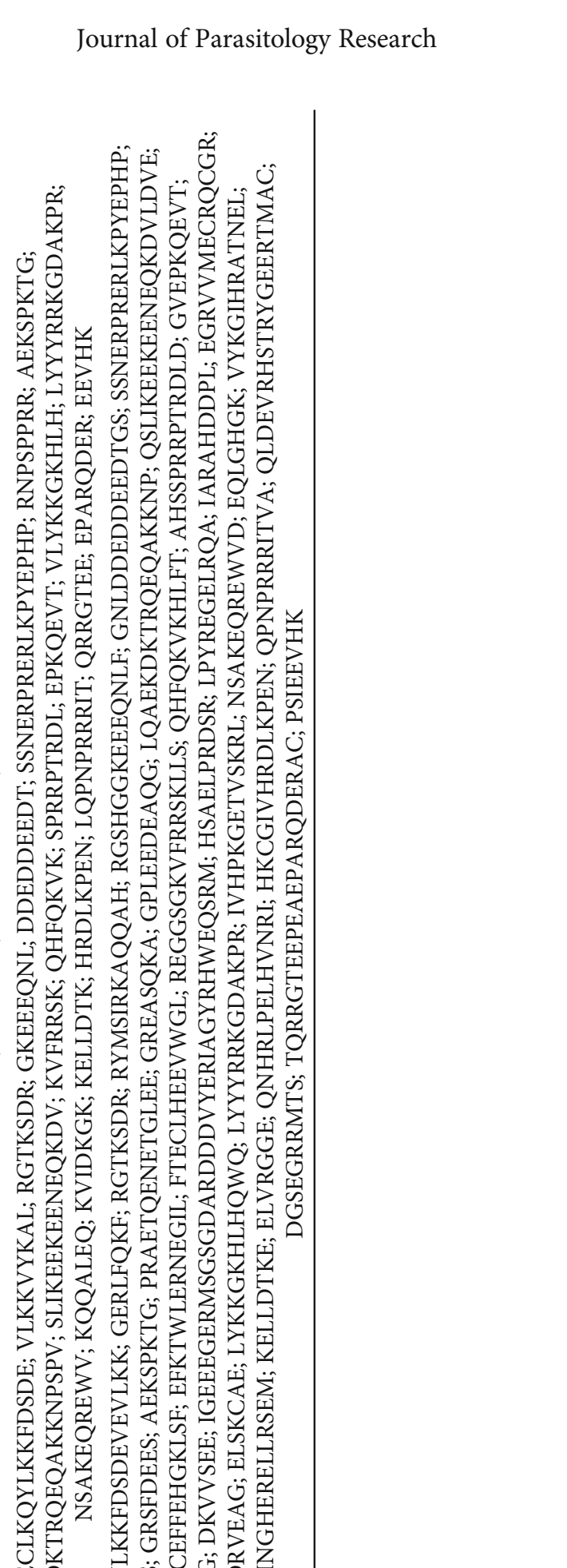

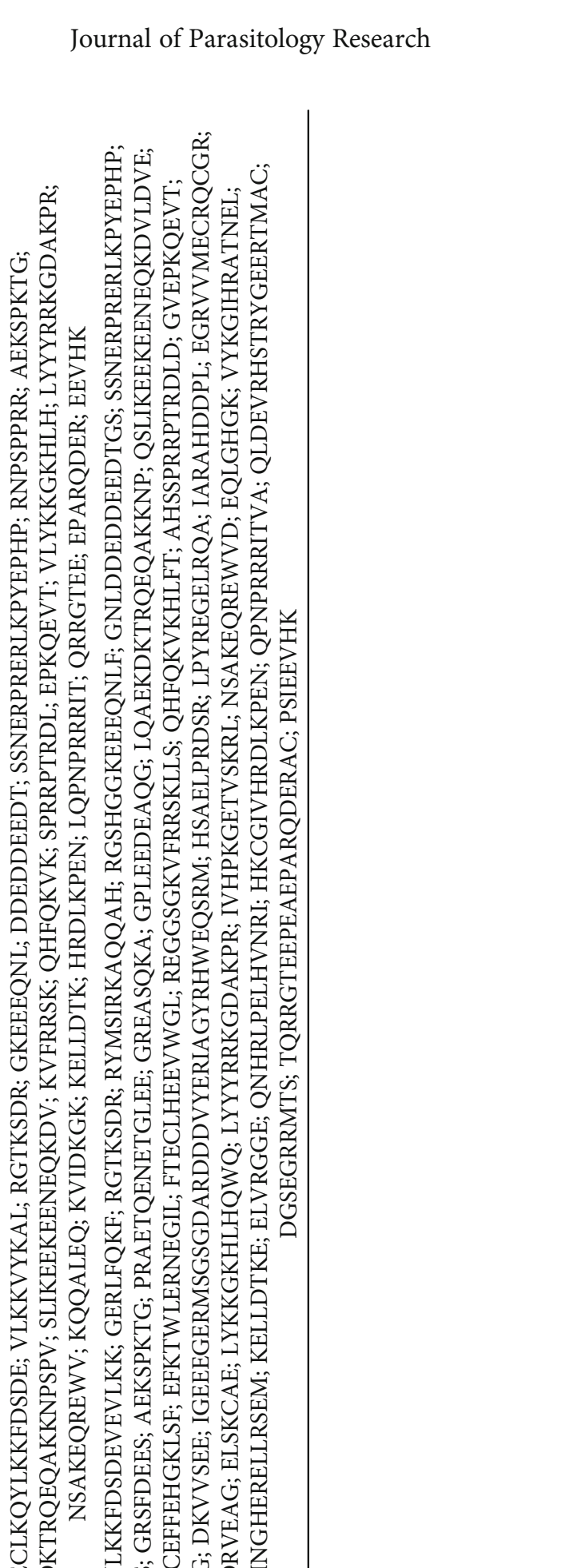

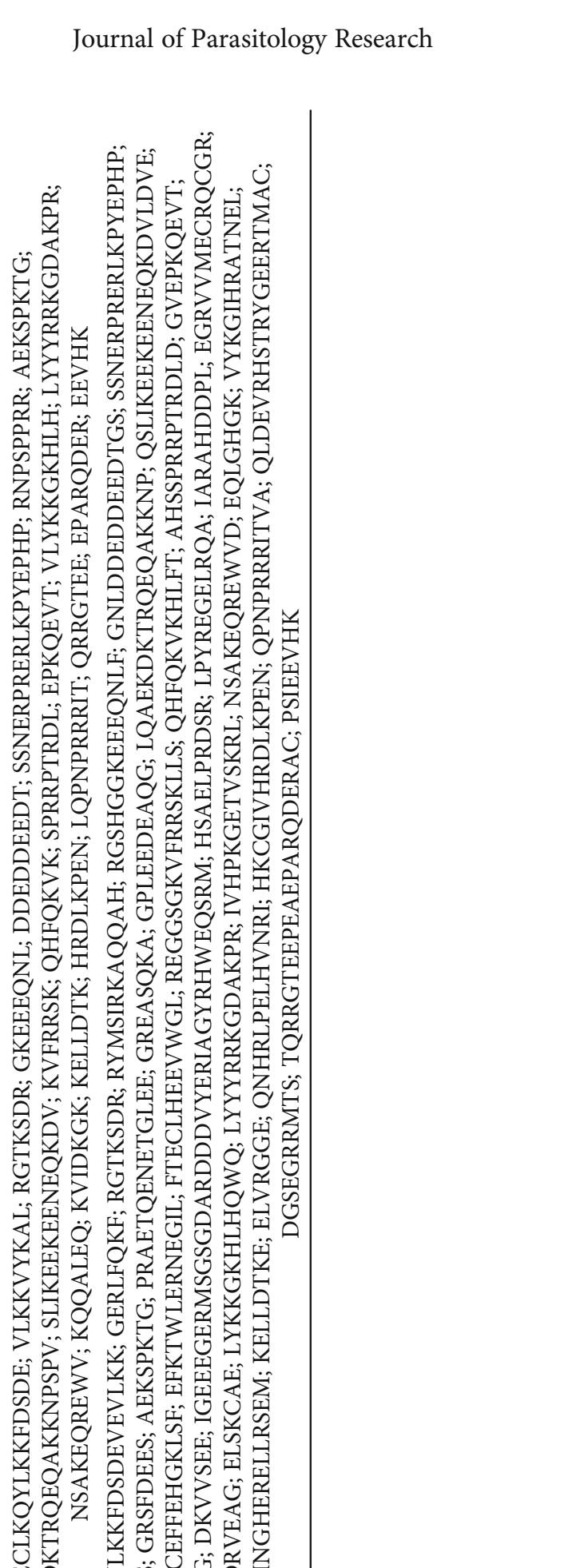

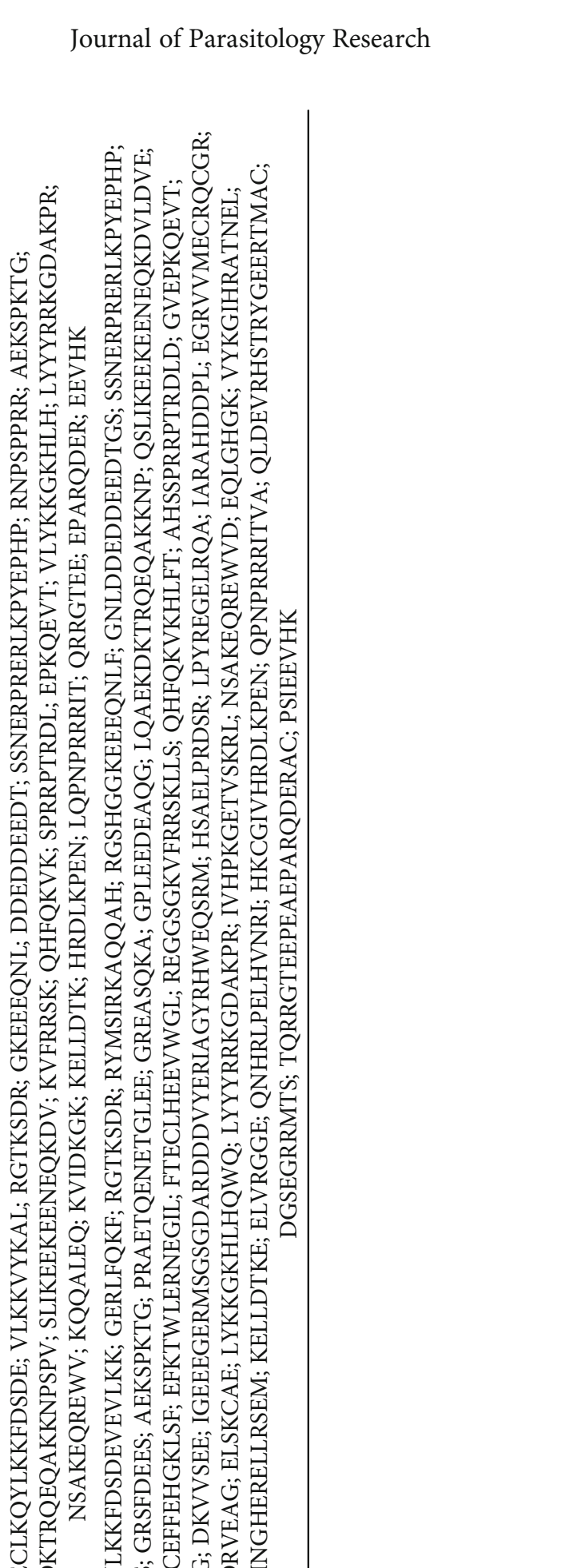

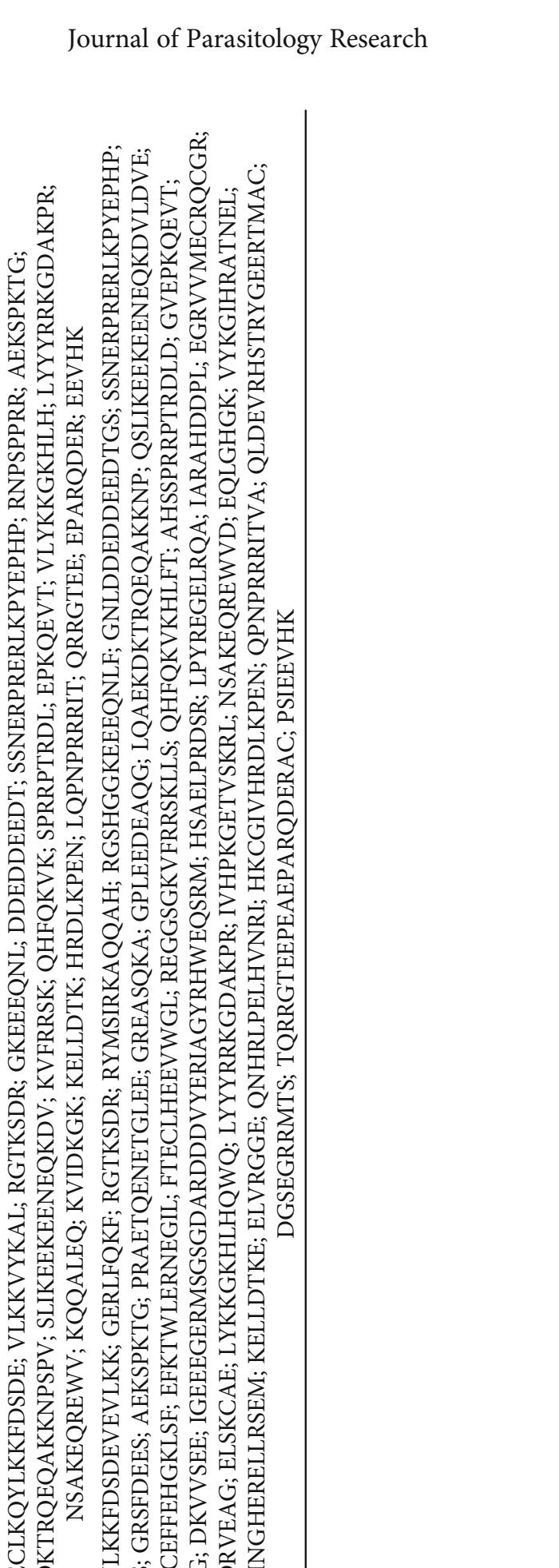

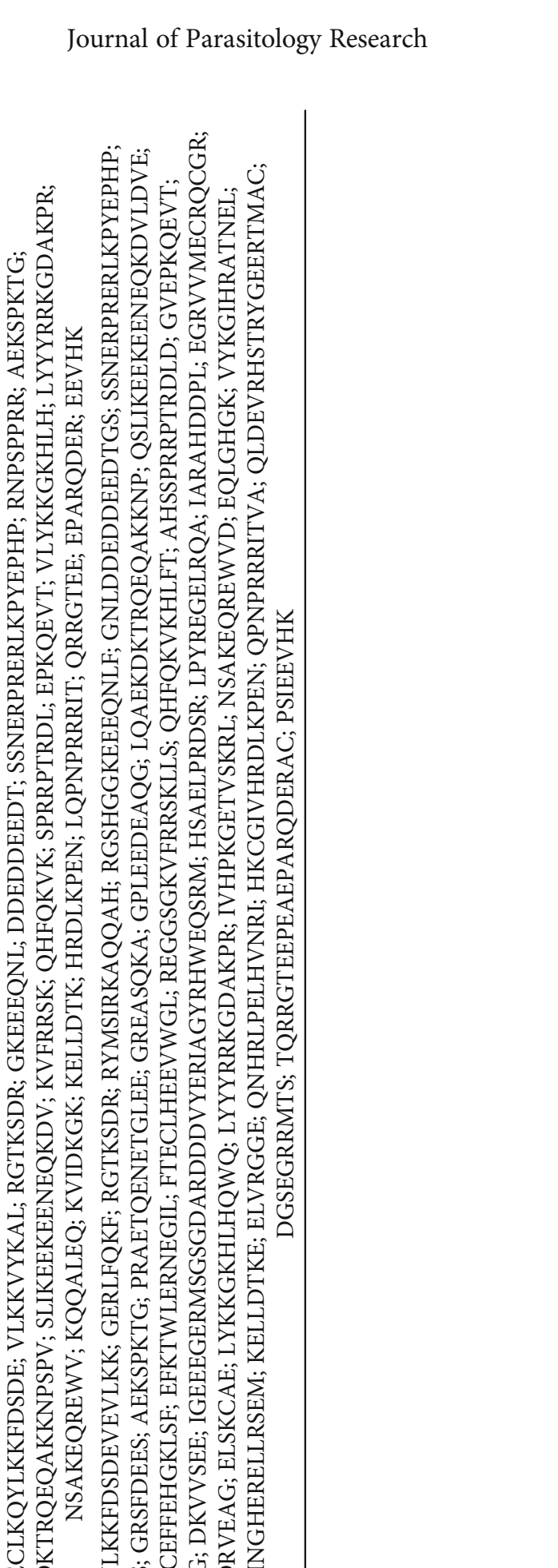

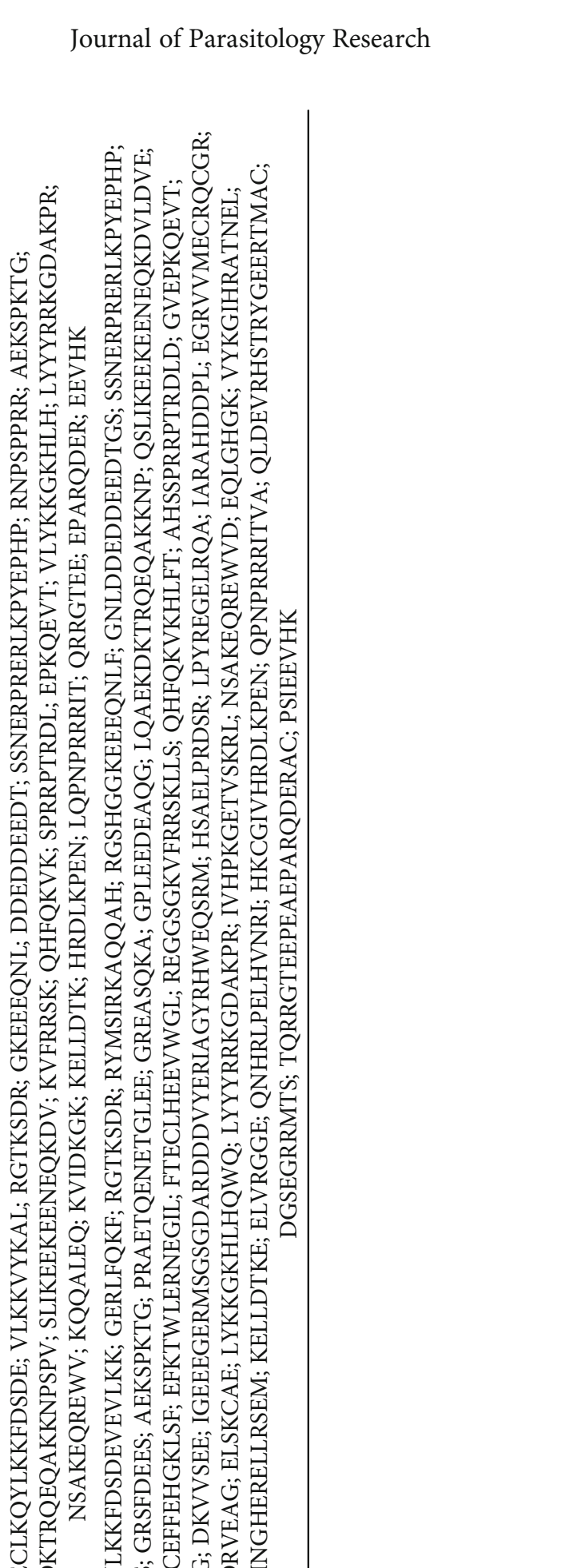

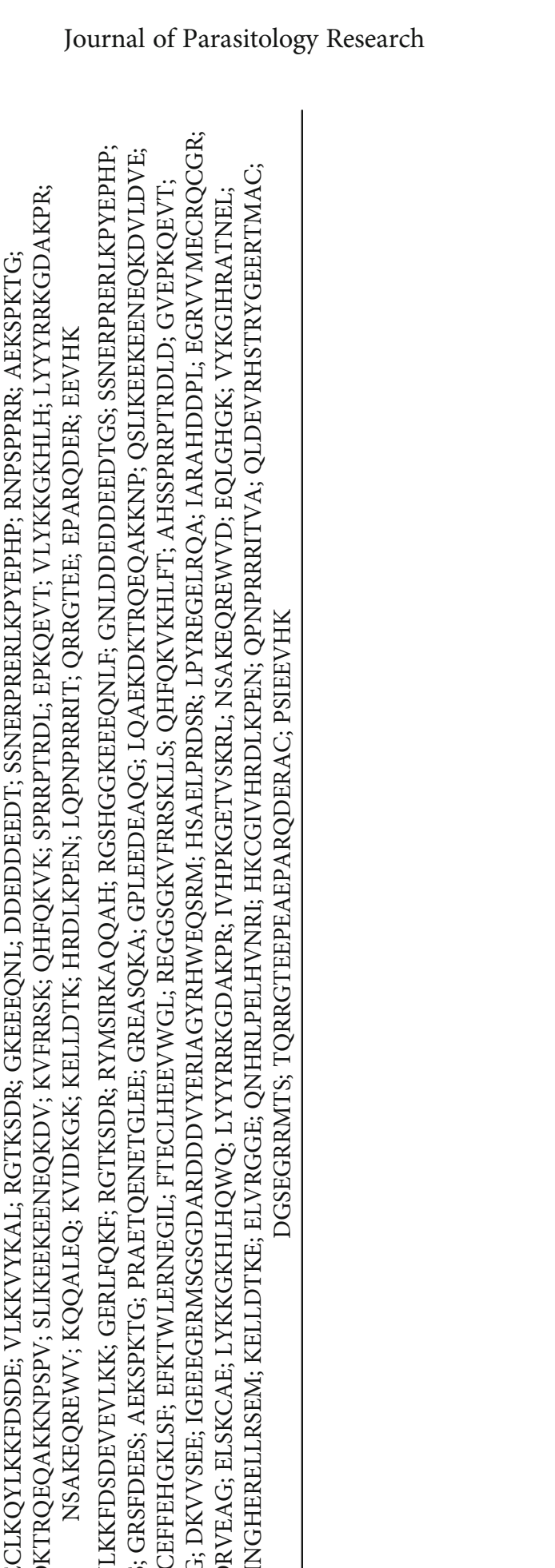

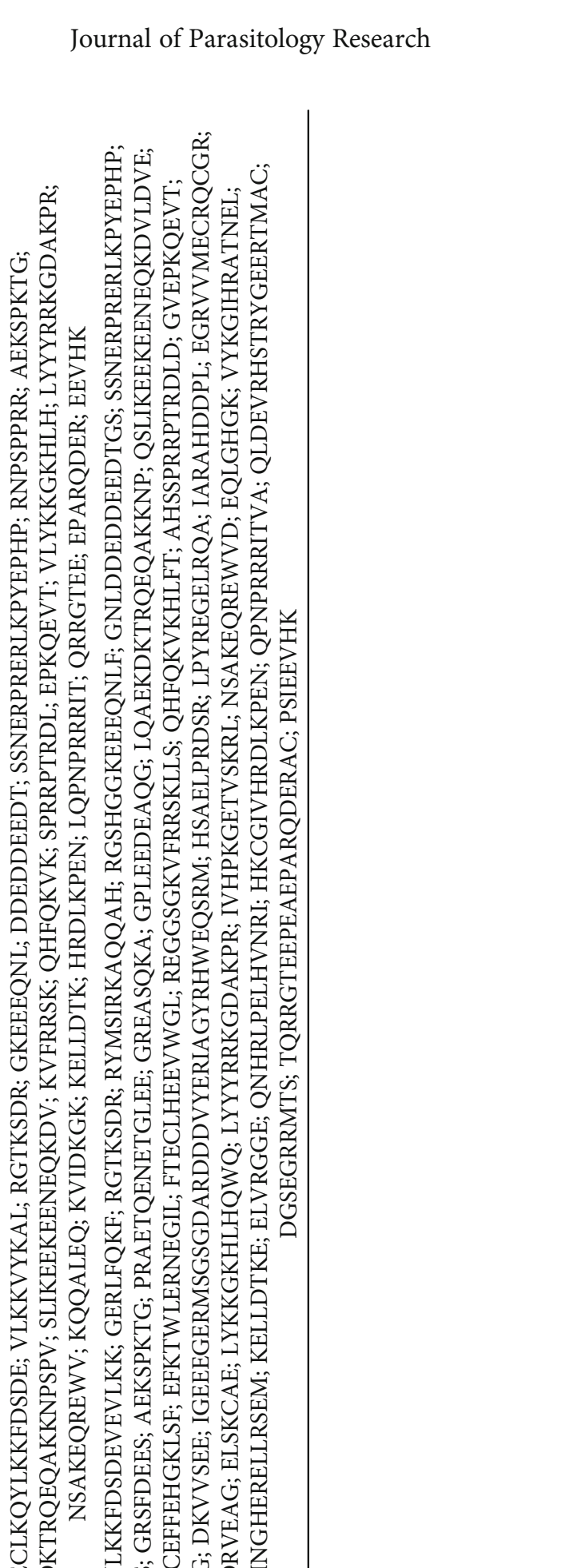

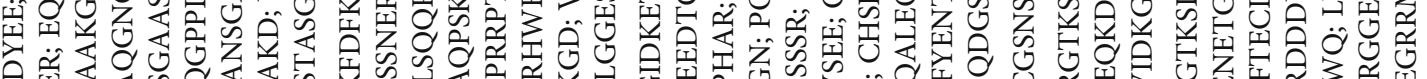

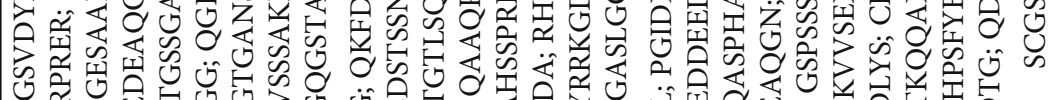

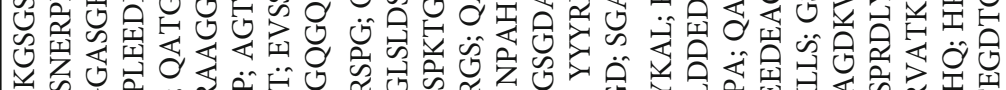

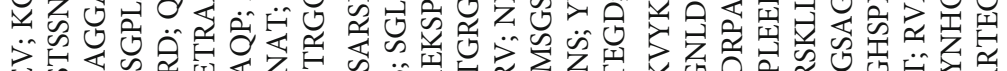

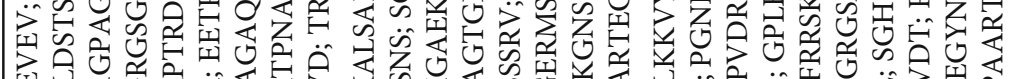

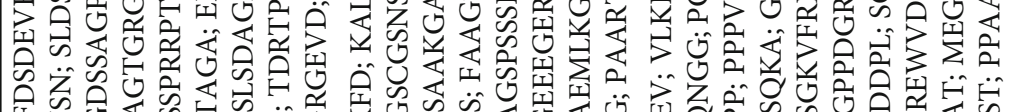

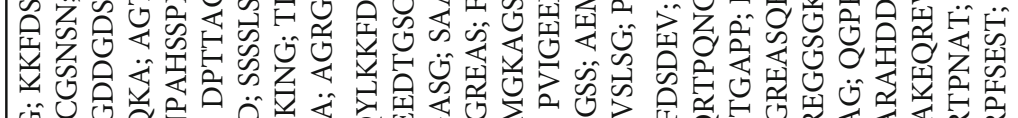

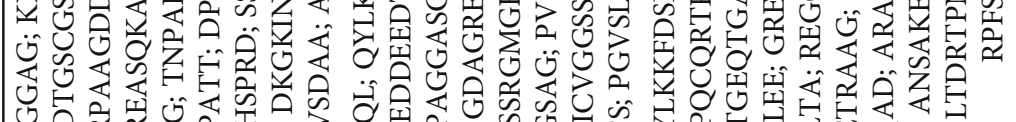

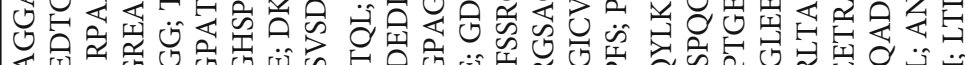

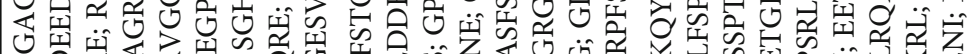

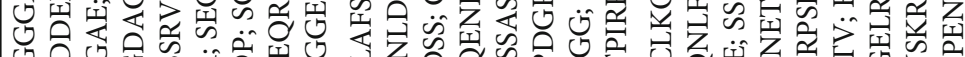

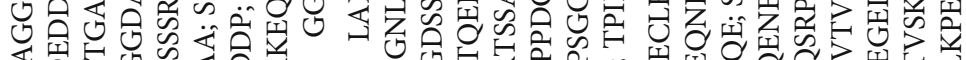

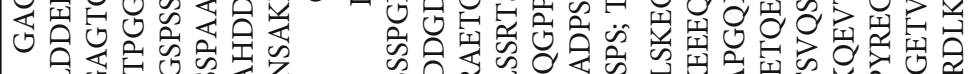

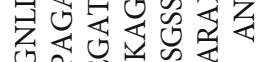

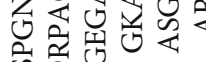
के

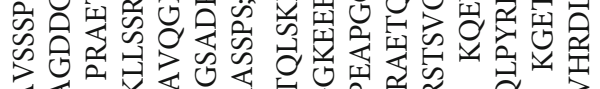

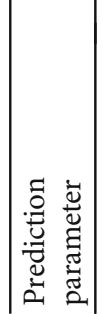
作

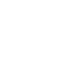

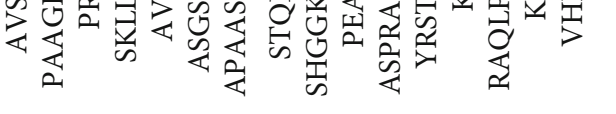

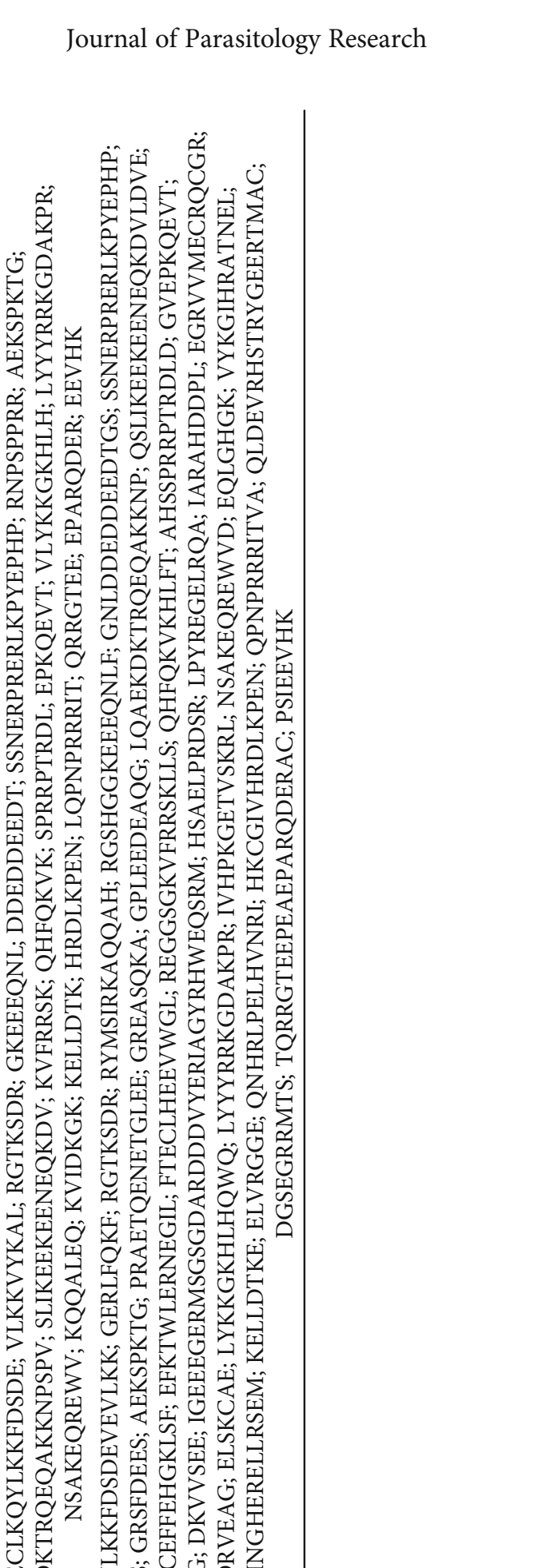

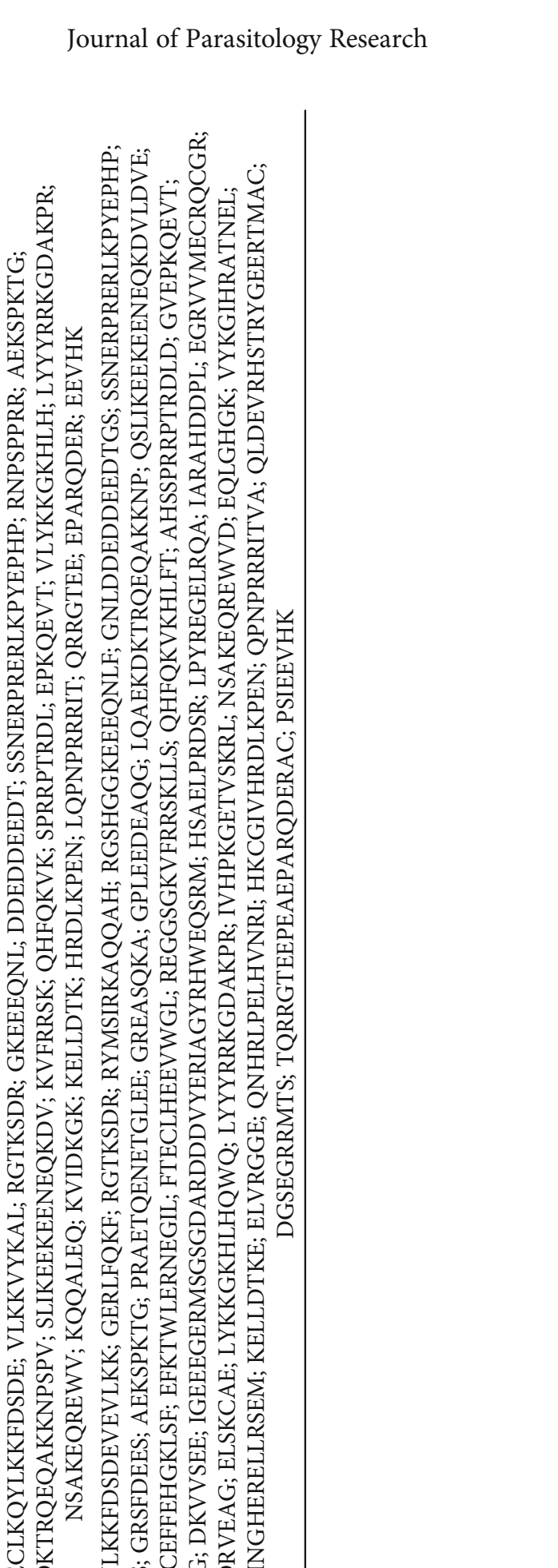

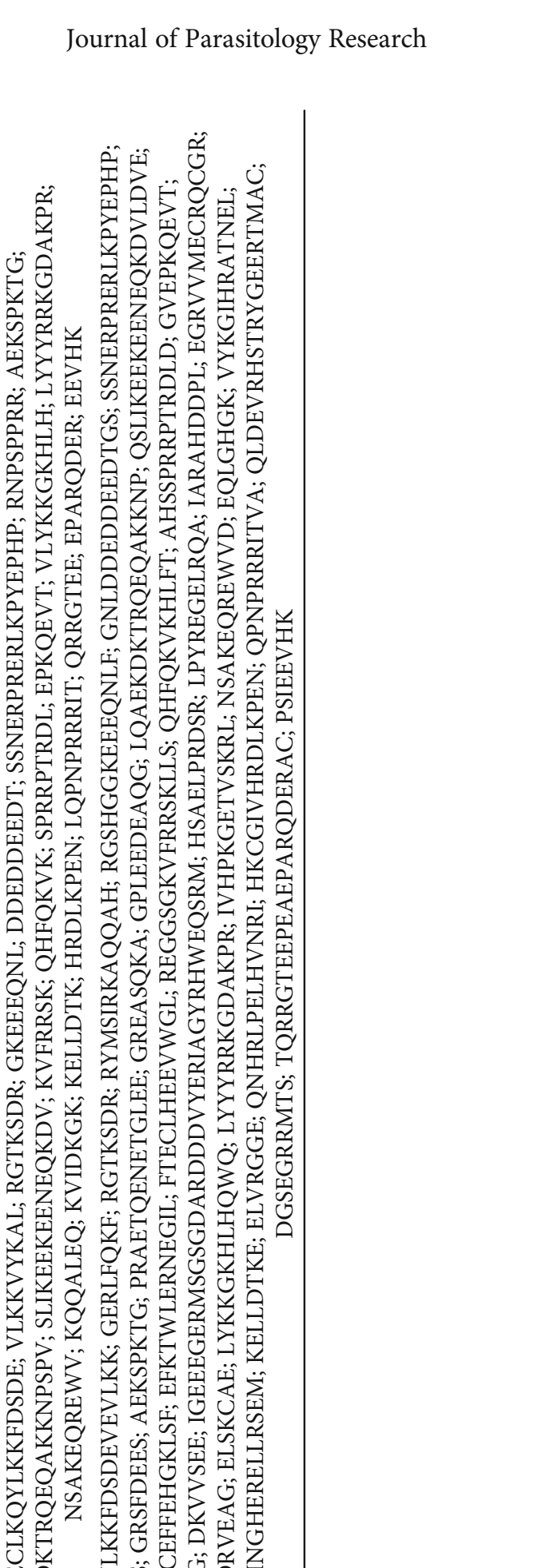

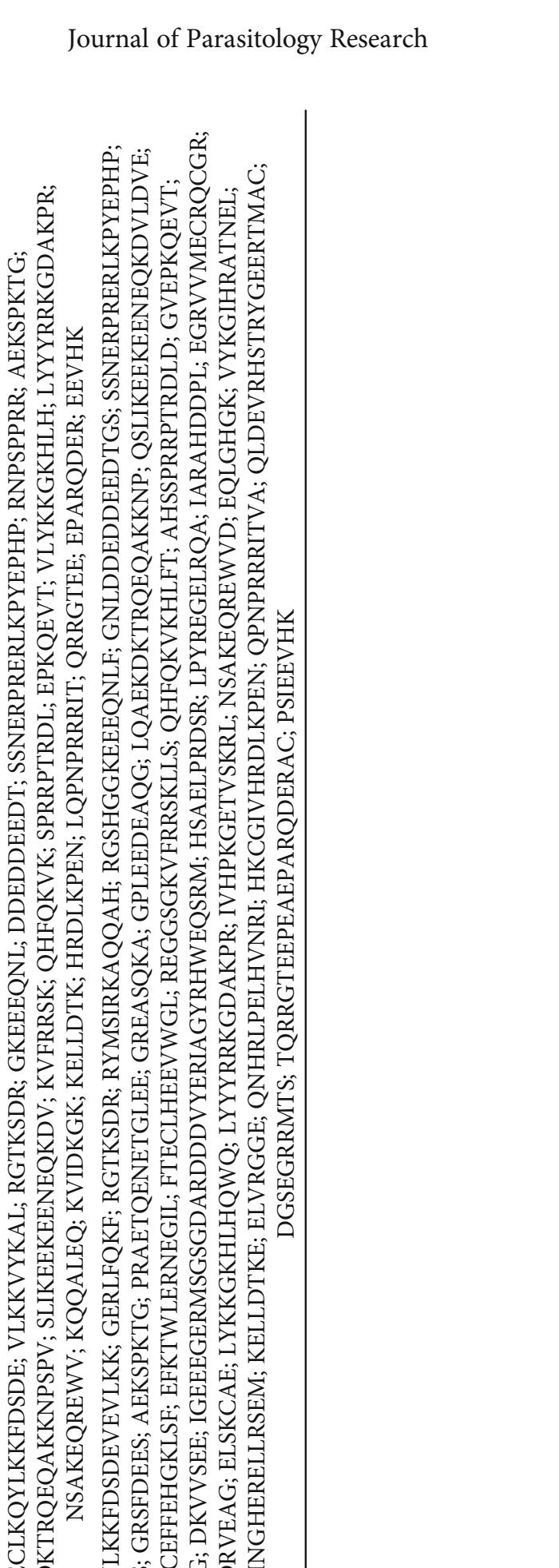

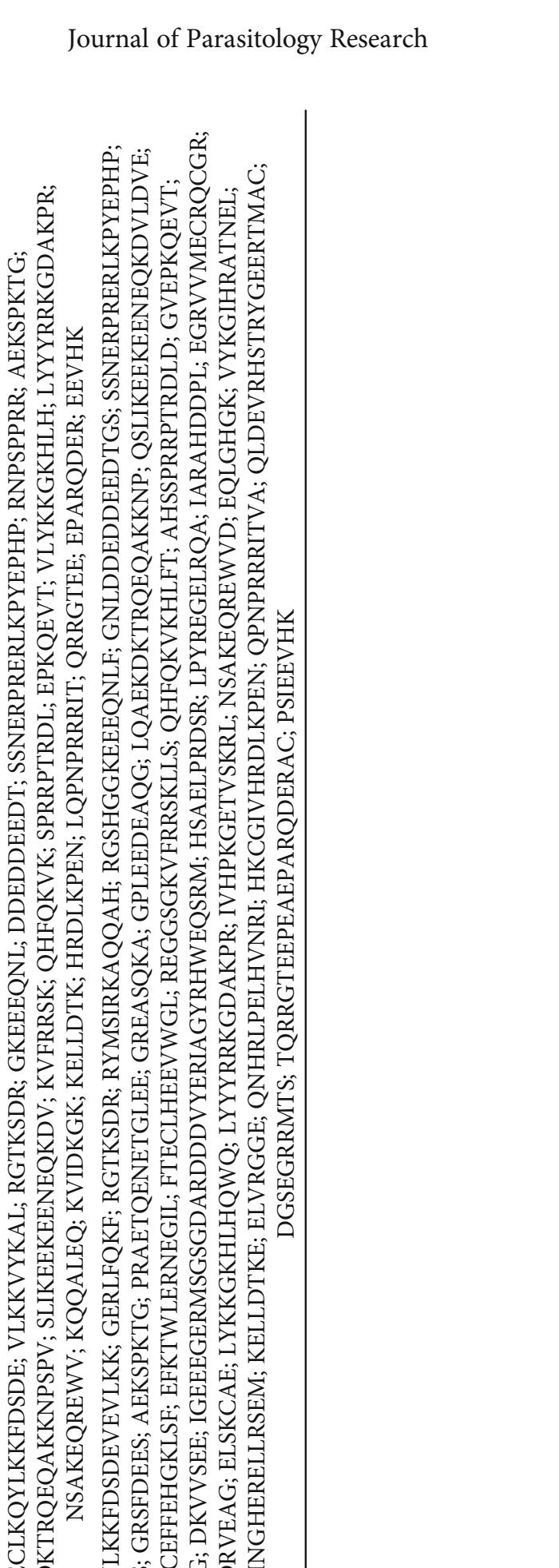

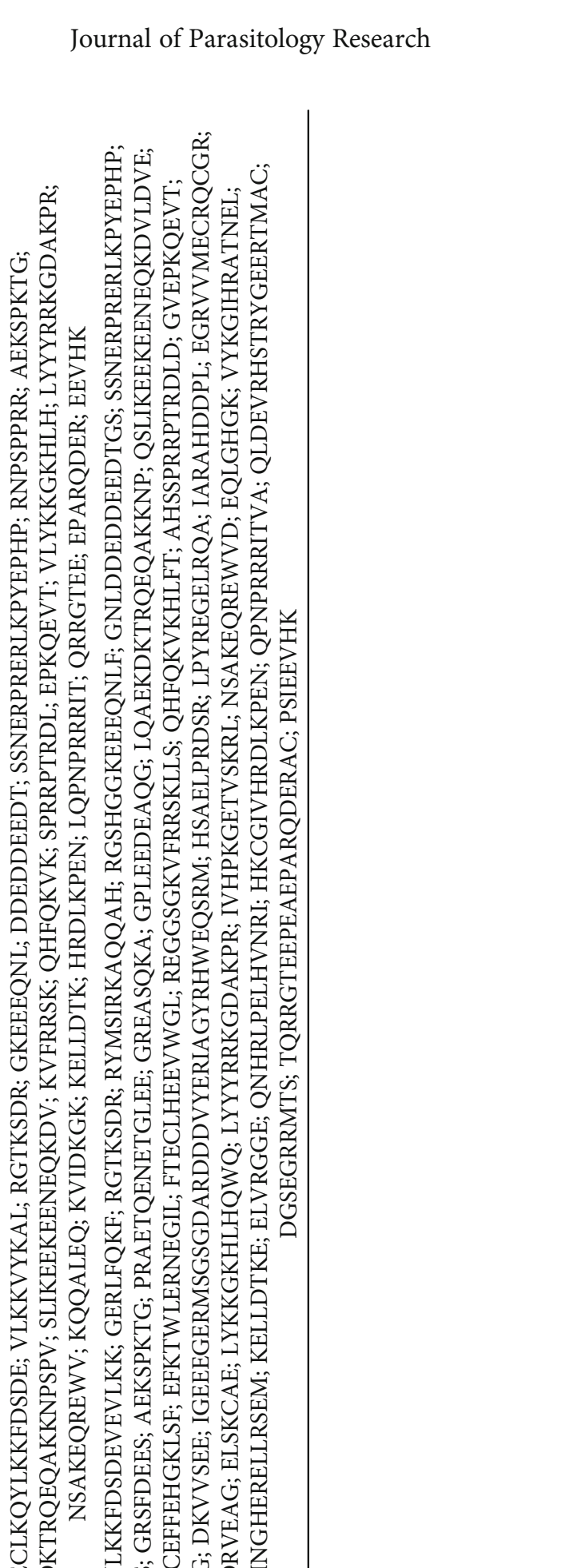

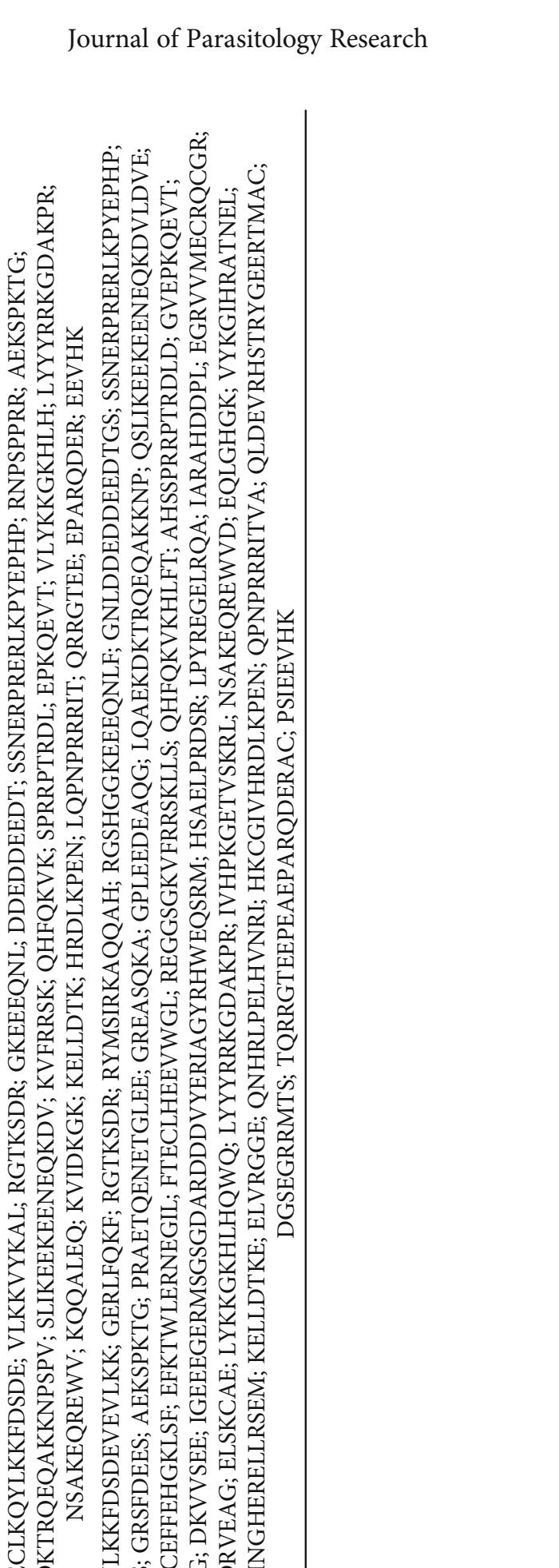

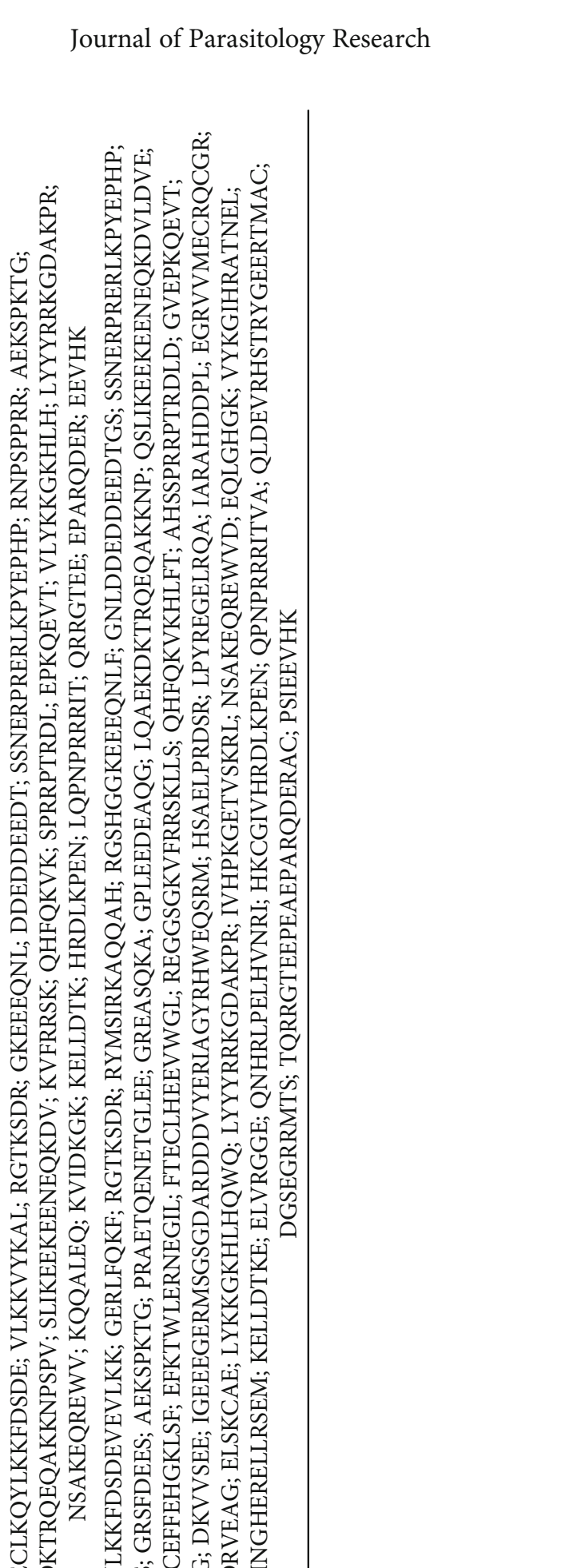

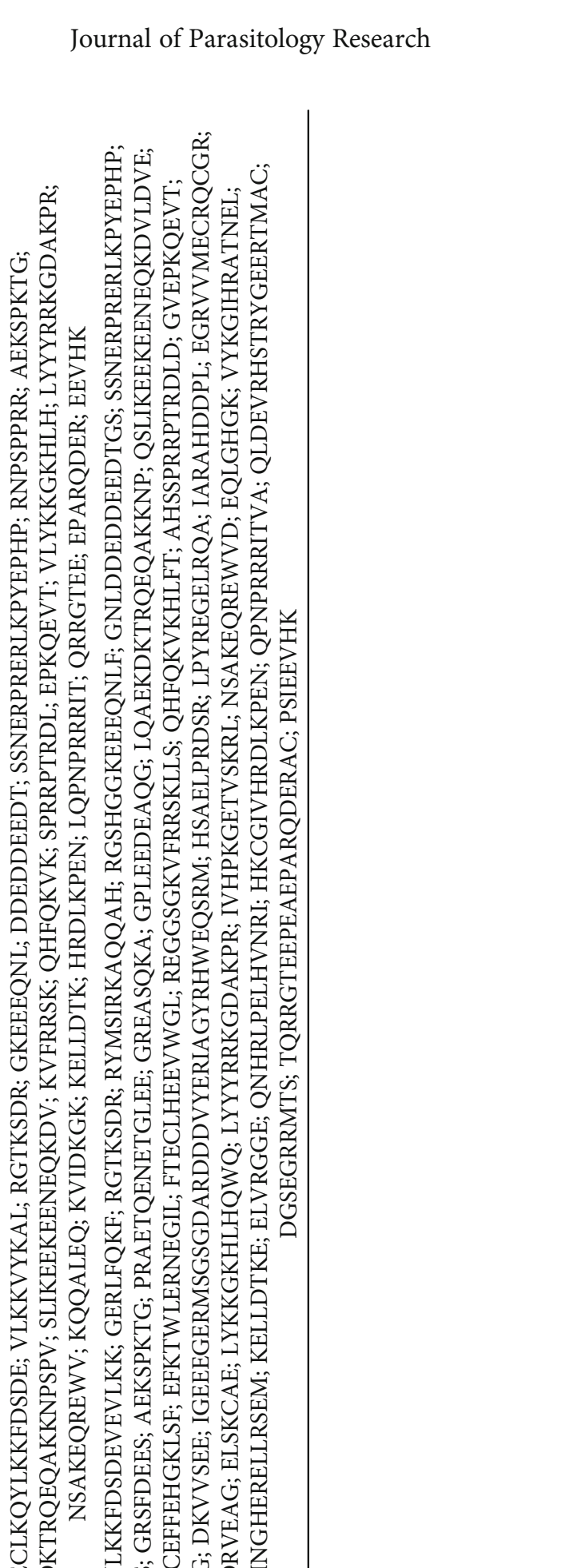

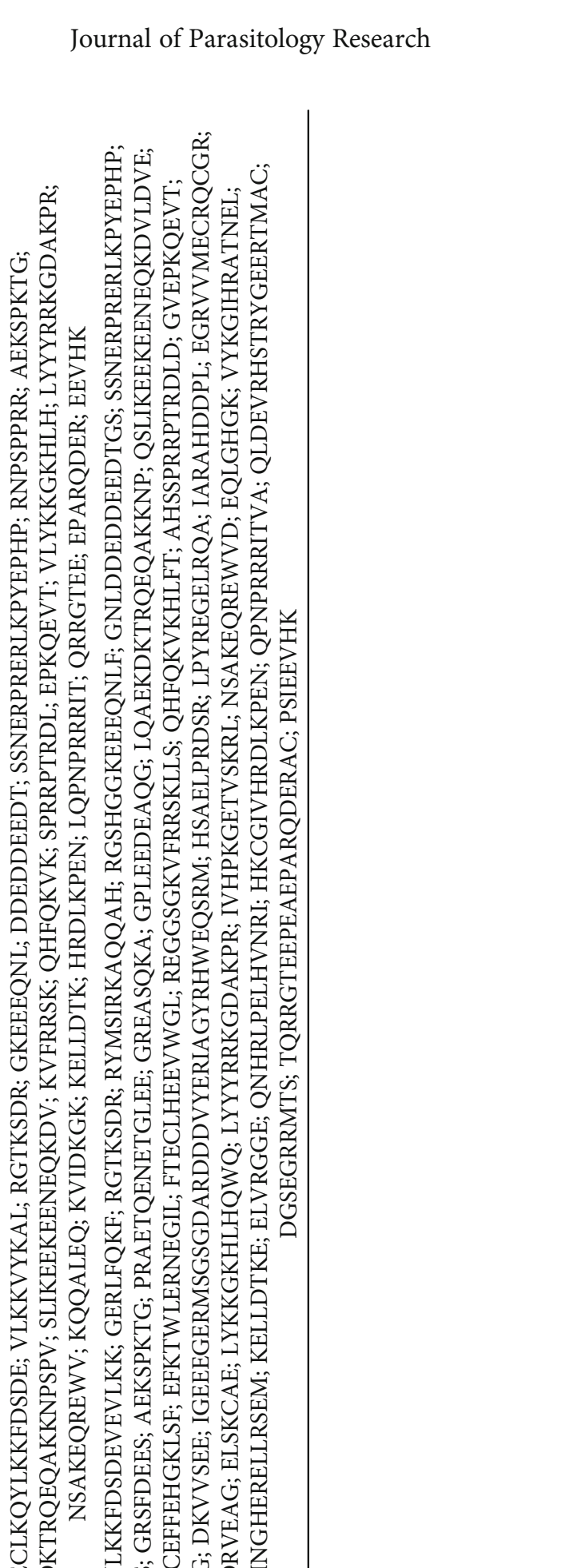

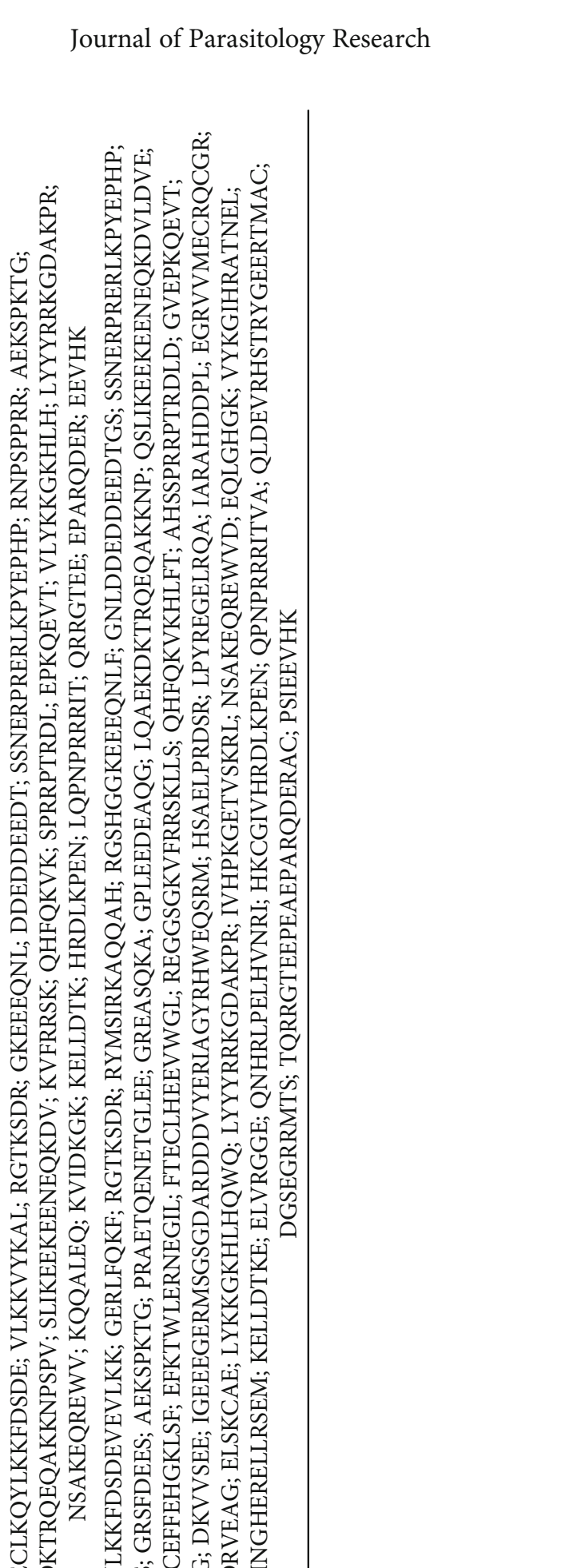

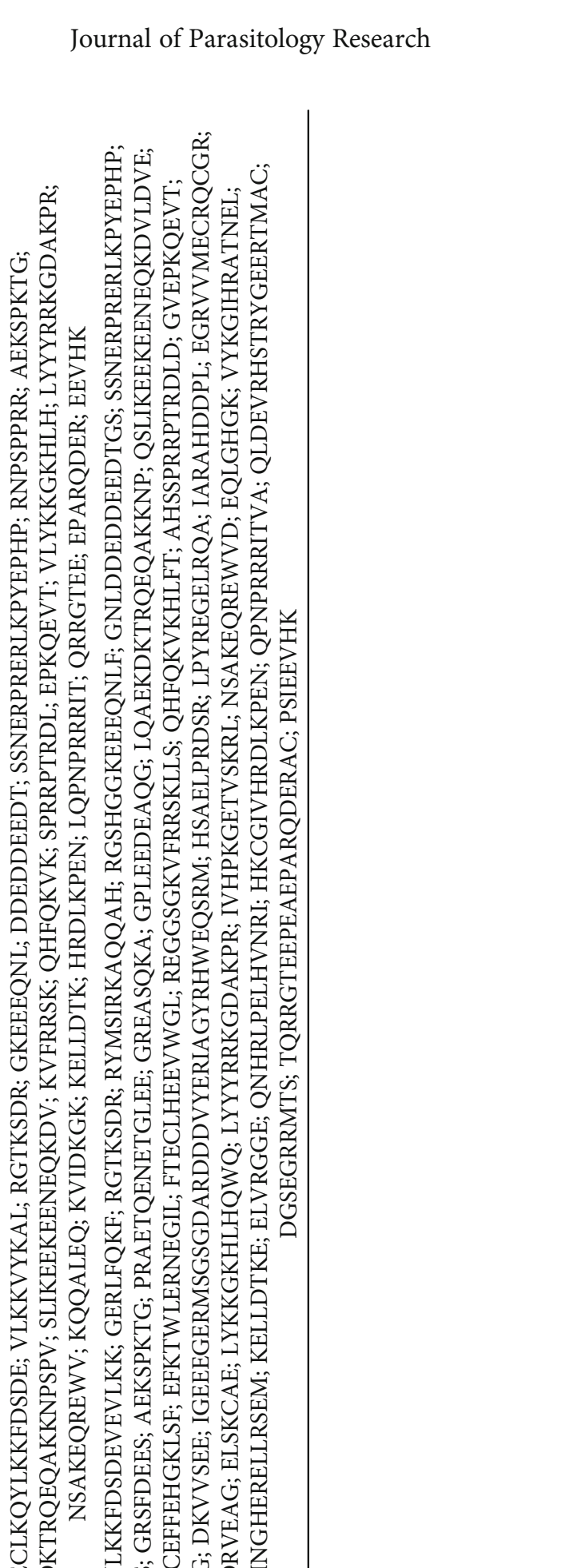

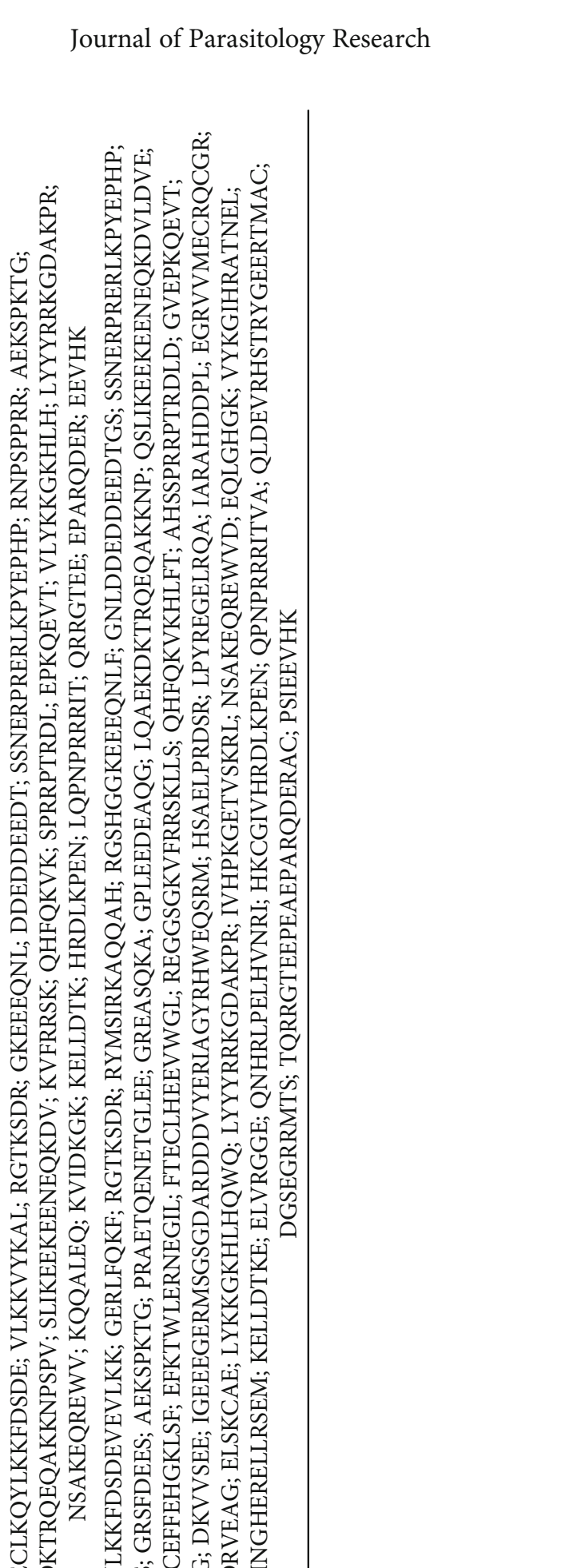

要

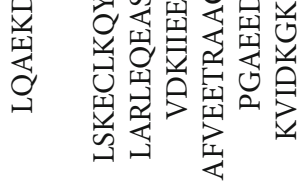




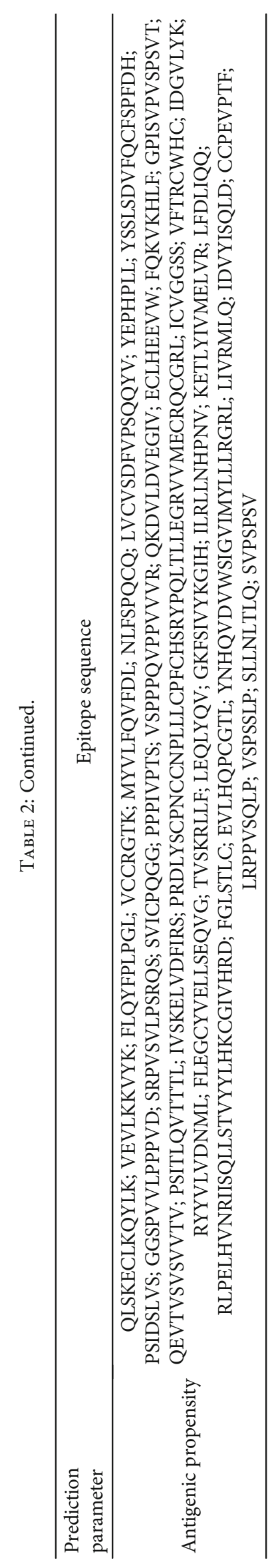


TABLE 3: The predicted B-cell epitopes via the ABCpred tool.

\begin{tabular}{|c|c|c|c|}
\hline Rank & Sequence & Start position & Score \\
\hline 1 & SSPPGTPASVVSPAAGAGPI & 965 & 0.95 \\
\hline 1 & EVPQAAQPSKGPTKSAMLLQ & 638 & 0.95 \\
\hline 1 & GGVSPPPQVPPVVVRAASPR & 564 & 0.95 \\
\hline 1 & GETVSKRLLFANSAKEQREW & 1497 & 0.95 \\
\hline 2 & DVLDVEGIVDKIIEECEFFE & 691 & 0.94 \\
\hline 2 & EEDEAQGNGMLEVPQAAQPS & 627 & 0.94 \\
\hline 2 & GAPTSVATPVAVSISSAPPA & 1922 & 0.94 \\
\hline 3 & KNGAKPLQNHGAPVATAGPP & 1839 & 0.93 \\
\hline 4 & ALEQLYQVGEQLGHGKFSIV & 1528 & 0.92 \\
\hline 5 & GAPSLAVGGATPLAGTTPPP & 927 & 0.91 \\
\hline 5 & FGYSASGGMIVNMQHFQKVK & 821 & 0.91 \\
\hline 5 & KDKTRQEQAKKNPSPVAQSL & 660 & 0.91 \\
\hline 5 & EDTGSCGSNSNFPGAQAQGA & 217 & 0.91 \\
\hline 5 & LPAAPAVASRAPAASSPSSL & 1868 & 0.91 \\
\hline 5 & DVWSIGVIMYLLLRGRLPFP & 1714 & 0.91 \\
\hline 5 & VYKGIHRATNELYAIKVIDK & 1547 & 0.91 \\
\hline 5 & SAKEQREWVDTLRVATKQQA & 1509 & 0.91 \\
\hline 6 & TPVYAVPAASAPGVSLSGGG & 1898 & 0.90 \\
\hline 6 & LIQQNHRLPELHVNRIISQL & 1620 & 0.90 \\
\hline 6 & SGDARDDDVYERIAGYRHWE & 1184 & 0.90 \\
\hline 6 & VAGAPTSSAGVEPKQEVTVS & 1003 & 0.90 \\
\hline 7 & QATGSSGAASAAAGASSVSA & 877 & 0.89 \\
\hline 7 & RSKLLSSRTSSASFSSRGMG & 789 & 0.89 \\
\hline 7 & VGSAHANAPPPGSGTPAPPP & 532 & 0.89 \\
\hline 7 & LYYYRRKGDAKPRGFMFLEG & 1454 & 0.89 \\
\hline 8 & AGALAVASPVSGAPSLAVGG & 916 & 0.88 \\
\hline 8 & PAAGDDGDSSAGPAGGASGE & 424 & 0.88 \\
\hline 8 & SEAAIDGVLYKKGKHLHQWQ & 1424 & 0.88 \\
\hline 9 & KNPSPVAQSLIKEEKEENEQ & 670 & 0.87 \\
\hline 9 & GGDAGREASQKAFAAGTGRG & 603 & 0.87 \\
\hline 9 & SAGPAGGASGESAAKGAEKS & 433 & 0.87 \\
\hline 9 & ASRNPSPPRRVSAQQPTHVG & 328 & 0.87 \\
\hline 9 & PHPLLARLEQEASSSEGYGR & 281 & 0.87 \\
\hline 9 & SSNERPRERLKPYEPHPLLA & 267 & 0.87 \\
\hline 9 & TPAEVPAGSPGGPSPSIEEV & 2112 & 0.87 \\
\hline 9 & SSLSDAGAQPAAGTGANSGA & 1351 & 0.87 \\
\hline 9 & KQEVTVSVSVVTVAGGGAGS & 1016 & 0.87 \\
\hline 10 & STSVQSRPSRLTAAGLQGIF & 752 & 0.86 \\
\hline 10 & LIKEEKEENEQKDVLDVEGI & 679 & 0.86 \\
\hline 10 & LQASPHARPAAGDDGDSSAG & 416 & 0.86 \\
\hline 10 & AQAQGAYPEAALVCVSDFVP & 231 & 0.86 \\
\hline 10 & GEERTMACCPEVPTFTIPKN & 1821 & 0.86 \\
\hline 10 & RCWHCGWELSKCAEMLKGNS & 1405 & 0.86 \\
\hline 10 & MSPQLAVDIVSKELVDFIRS & 1207 & 0.86 \\
\hline 10 & VASGSSPAAPGVTGVTEAVA & 1044 & 0.86 \\
\hline 11 & TQGAPGSPPVRGFSGGGGSR & 488 & 0.85 \\
\hline 11 & KTASRFTSAIKRTFSTQSSS & 468 & 0.85 \\
\hline 11 & EESSGASSYSSLSDVFQCFS & 304 & 0.85 \\
\hline
\end{tabular}

TABle 3: Continued.

\begin{tabular}{|c|c|c|c|}
\hline Rank & Sequence & Start position & Score \\
\hline 11 & GSPGVSGALLSPAAGSKSVP & 2042 & 0.85 \\
\hline 11 & GDKVVSEEAFPVIGEEEGER & 1161 & 0.85 \\
\hline 12 & ISVPVSPSVTAVATAAVTQV & 984 & 0.84 \\
\hline 12 & NMQHFQKVKHLFTNPAHSSP & 832 & 0.84 \\
\hline 12 & ASQKAFAAGTGRGSGPLEED & 610 & 0.84 \\
\hline 12 & VEETRAAGGATAPGTSVTHT & 1125 & 0.84 \\
\hline 12 & TVAGGGAGSETQPAMASVAS & 1027 & 0.84 \\
\hline 13 & DKIIEECEFFEHGKLSFPEF & 700 & 0.83 \\
\hline 13 & PGIDKETFLQYFPLPGLWGE & 64 & 0.83 \\
\hline 13 & GYRHWEQSRMSPQLAVDIVS & 1198 & 0.83 \\
\hline 13 & TTGATTAVGGPVSEGPATTP & 1072 & 0.83 \\
\hline 14 & QTGPSGPSSPPGTPASVVSP & 958 & 0.82 \\
\hline 14 & GTLSQQPRGGITKTASRFTS & 456 & 0.82 \\
\hline 14 & AGAVSSSPGNLDDEDDEEDT & 200 & 0.82 \\
\hline 14 & PCGTLAYVAPEVLTMEGYNH & 1692 & 0.82 \\
\hline 14 & NSVGRGSHGGKEEEQNLFSP & 166 & 0.82 \\
\hline 14 & LLSTVYYLHKCGIVHRDLKP & 1639 & 0.82 \\
\hline 14 & GFAIVHPKGETVSKRLLFAN & 1489 & 0.82 \\
\hline 14 & GFMFLEGCYVELLSEQVGGR & 1467 & 0.82 \\
\hline 14 & CRQCGRLGGSSSSLSDAGAQ & 1340 & 0.82 \\
\hline 14 & LSGASGPGSGALASPSEGAS & 1249 & 0.82 \\
\hline 14 & KELVDFIRSSHQSLHSAELP & 1218 & 0.82 \\
\hline 14 & ATAAAAAFVEETRAAGGATA & 1117 & 0.82 \\
\hline 14 & AGAAAAAATAAAAAFVEETR & 1110 & 0.82 \\
\hline 15 & DPATPAPQPSRLSSSPQMQA & 859 & 0.81 \\
\hline 15 & FFEHGKLSFPEFKTWLERNE & 708 & 0.81 \\
\hline 15 & TGLEELGEGATPGGDAGREA & 591 & 0.81 \\
\hline 15 & APPAALSSRPSIDSLVSASS & 369 & 0.81 \\
\hline 15 & SPTGEQTGAPPAALSSRPSI & 361 & 0.81 \\
\hline 15 & EEPEAEPARQDERACGTPAE & 2096 & 0.81 \\
\hline 15 & PAARTEGDTGPVEGAAVSPS & 1940 & 0.81 \\
\hline 15 & KAQQAHSEGSNSVGRGSHGG & 156 & 0.81 \\
\hline 15 & VGGPVSEGPATTPSITLQVT & 1079 & 0.81 \\
\hline 16 & SGSVDYEEFLIGIAVCCRGT & 94 & 0.80 \\
\hline 16 & ICPQGGISPVGSAHANAPPP & 523 & 0.80 \\
\hline 16 & VQSTRVGAGGGAGGAGPANS & 4 & 0.80 \\
\hline 16 & ASSSEGYGRSFDEESSGASS & 292 & 0.80 \\
\hline 16 & GESVSDAAPVAGRGEVDLTR & 1978 & 0.80 \\
\hline 17 & PAPPPIVPTSSGGVPAPGGV & 547 & 0.79 \\
\hline 17 & GGGSRPVSVLPSRQSSEASV & 503 & 0.79 \\
\hline 17 & SATPPVAAEAGSPGVSGALL & 2032 & 0.79 \\
\hline 17 & NHGAPVATAGPPAALRPPVS & 1847 & 0.79 \\
\hline 17 & LFSPQCQRTPQNGGSSGTAG & 182 & 0.79 \\
\hline 17 & LLLRGRLPFPINQAFGHPSF & 1724 & 0.79 \\
\hline 17 & ELVRGGELFDLIQQNHRLPE & 1610 & 0.79 \\
\hline 17 & NELYAIKVIDKGKINGHERE & 1556 & 0.79 \\
\hline 17 & AAIARAHDDPLACSGHSPRD & 1286 & 0.79 \\
\hline 17 & EAVAVASVPGTPTTGATTAV & 1060 & 0.79 \\
\hline
\end{tabular}


TABle 3: Continued.

\begin{tabular}{lccc}
\hline Rank & Sequence & Start position & Score \\
\hline 18 & PSKGPTKSAMLLQAEKDKTR & 645 & 0.78 \\
18 & QEAPGTVSSPTGEQTGAPPA & 353 & 0.78 \\
18 & APGCSDLSSASSGTQRRGTE & 2077 & 0.78 \\
18 & ASPASLLNLTLQDGSEGRRM & 2011 & 0.78 \\
18 & DEVRHSTRYGEERTMACCPE & 1812 & 0.78 \\
18 & VHRDLKPENILLTDRTPNAT & 1652 & 0.78 \\
18 & GGASGSADPSGGPGAEEDRV & 1371 & 0.78 \\
19 & PSRQSSEASVICPQGGISPV & 513 & 0.77 \\
19 & AAVSPSSLPAGSLDEVPESG & 1954 & 0.77 \\
19 & YENTPVSFDGAVWREVSSSA & 1744 & 0.77 \\
19 & ETLYIVMELVRGGELFDLIQ & 1603 & 0.77 \\
19 & LLSEQVGGRQYGFAIVHPKG & 1478 & 0.77 \\
19 & GASARAQLPYREGELRQADL & 1266 & 0.77 \\
20 & PLPGLWGERLFQKFDFKGSG & 76 & 0.76 \\
20 & PRAETQENETGLEELGEGAT & 582 & 0.76 \\
20 & FGLSTLCAPNEVLHQPCGTL & 1677 & 0.76 \\
20 & ATIKLTDFGLSTLCAPNEVL & 1670 & 0.76 \\
20 & KHLHQWQARYYVLVDNMLYY & 1437 & 0.76 \\
20 & CSGHSPRDLYSCPNCCNPLL & 1298 & 0.76 \\
20 & AGGGAGGAGPANSLAFSTQL & 11 & 0.76 \\
21 & GREGGSGKVFRRSKLLSSRT & 778 & 0.75 \\
21 & VCVSDFVPSQQYVATGSGLS & 243 & 0.75 \\
21 & AVSISSAPPAARTEGDTGPV & 1932 & 0.75 \\
21 & KNIDVYISQLDEVRHSTRYG & 1802 & 0.75 \\
21 & ELVAMLSNLPNLDRYMSIRK & 137 & 0.75 \\
21 & REGELRQADLAAIARAHDDP & 1276 & 0.75 \\
21 & VFDLNSDGYIQKSELVAMLS & 124 & 0.75 \\
21 & TAPGTSVTHTATATAVQGPP & 1135 & 0.75 \\
\hline & & &
\end{tabular}

sites (Table 1) were recognized in the CDPK7, representing that the CDPK7 sequence is composed of 299 possible PTM sites.

3.3. Transmembrane Domains and Subcellular Location. Based on the TMHMM output, no transmembrane domain was found for CDPK7 (Figures 2(a) and 2(b)). Moreover, by PSORT II, the CDPK7 subcellular site was predicted as follows: $78.3 \%$ nuclear, $8.7 \%$ cytoplasmic, $8.7 \%$ plasma membrane, and $4.3 \%$ cytoskeletal.

3.4. Secondary and Tertiary Structures. The secondary structure of CDPK7 was predicted via the GOR4 online server, suggesting 502 alpha-helix, 320 extended strands, and 1311 random coils (Figures 3(a) and 3(b)). Moreover, the SWISS-MODEL analysis is shown in Figures 4(a)-4(d).

3.5. Refinement and Validation of Tertiary Structure. Protein validation by means of the SWISS-MODEL server displayed that $92.86 \%$ of residues were situated in favored regions and $1.65 \%$ in the outlier regions. According to the Ramachandran plot, there were $97.80 \%$ residues in the favored region with $0.27 \%$ residues in the outlier regions of the refined model (Figure 5).

3.6. Predicted Linear and Discontinuous B-Cell Epitopes of the CDPK7 Protein. The predicted linear B-cell epitopes by the Bcepred are listed in Table 2. The outputs of the ABCpred server are tabulated in Table 3 (only the epitopes over scores of 0.75 are embedded in Table 3). The higher peptide score proposes the greater chance of being an epitope. The present server estimated 124 epitopes over 0.75 scores on the sequence, in which the linear epitope SSPPGTPASVVSPAAGAGPI (score: 0.95 ) had the greatest score. Four epitopes with over 0.95 scores were as follows: "SSPPGTPASVVSPAAGAGPI," "EVPQAAQPSKGPTKSAMLLQ," "GGVSPPPQVPPVVVRAASPR," and "GETVSKRLLFANSAKEQREW." The average score of antigenicity, beta-turn, flexibility, hydrophilicity, Bepipred linear epitope prediction, and surface accessibility for the CDPK7 protein using the IEDB online server was 1.026, 1.042, $1.017,2.396,0.350$, and 1.00, respectively (Figure 6). Five discontinuous B-cell epitopes were predicted using the ElliPro server (Table 4).

3.7. MHC-Binding Epitopes. The results are listed in Tables 5 and 6. Epitopes were assessed regarding antigenicity, and those highly antigenic epitopes were finally selected.

3.8. CTL Epitope Prediction. The high-ranked CTL epitopes predicted by the CTLpred tool for CDPK7 protein are summarized in Table 7. Epitopes were assessed regarding antigenicity, and those highly antigenic epitopes were finally selected.

3.9. Antigenic and Allergenic Profiles. The antigenic profile of CDPK7 was conducted by the VaxiJen web server with score of 0.7074 (threshold: 0.5). Based on AllergenFP and AllerTOP v2.0 analyses, the CDPK7 protein was appraised as possible nonallergen.

\section{Discussion}

Toxoplasmosis is a significant menace to human society as well as livestock industry $[2,8,60]$. Thus, the design and improvement of an efficient vaccine against $T$. gondii infection is still a great challenge for researchers against toxoplasmosis in domestic animals and humans [61]. Recently, bioinformatics tools are more focused for rational vaccine design, with some advantage, including the following: (i) time- and cost-effectiveness; (ii) accurately targeting, long-lasting immunity with favorable polarity in cellular components; and (iii) elimination of undesired responses through specific, epitope-based construct design. Nevertheless, the obtained in silico results are only theoretical data which must be confirmed using wet lab experiments inevitably [62].

It has been known that CDPK7 contributes to several functions in $T$. gondii such as gliding movement, host-cell invasion, and egress as well as other vital growth processes [34]. Here, we conducted a comprehensive analysis of TgCDPK7, a member of the CDPK family in T. gondii. The 


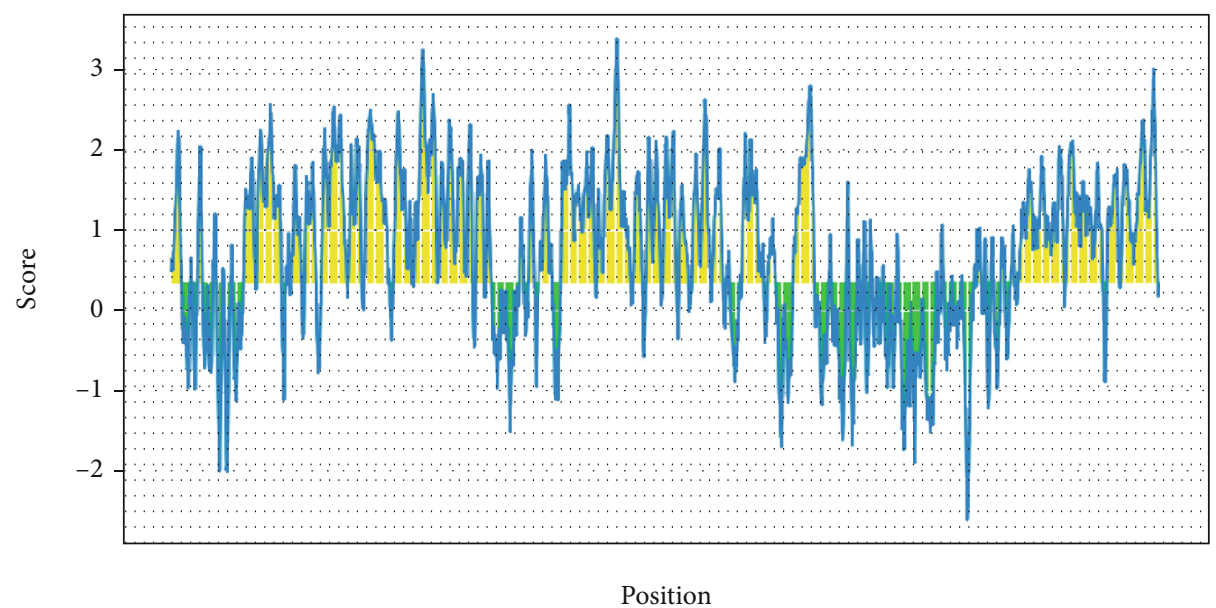

(a)

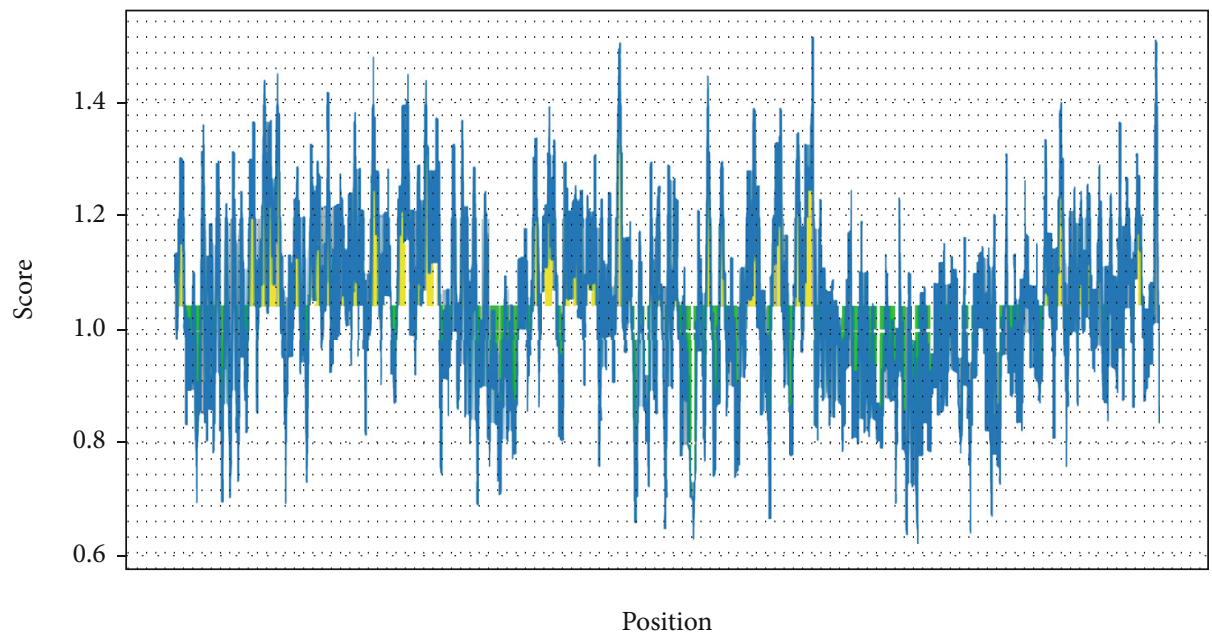

(b)

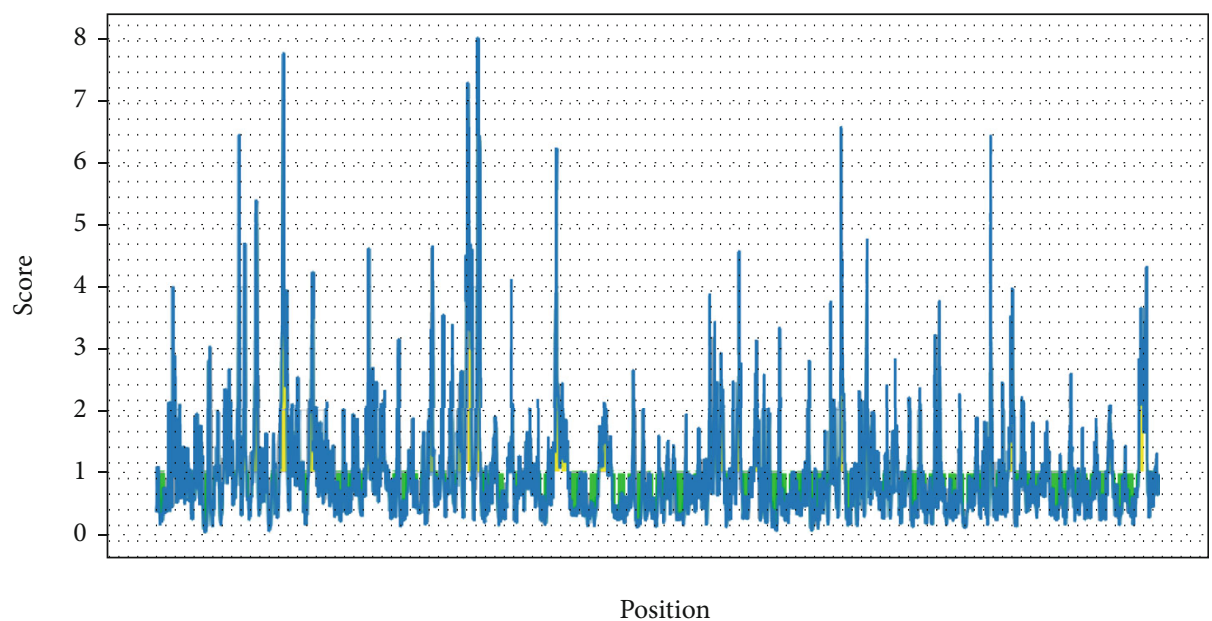

(c)

Figure 6: Continued. 


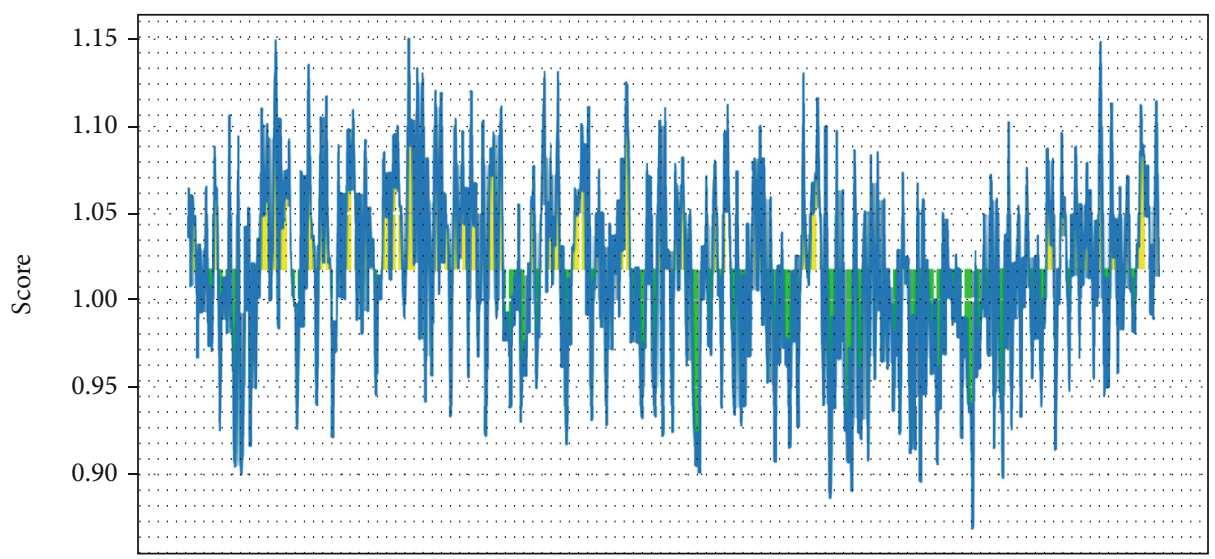

Position

(d)

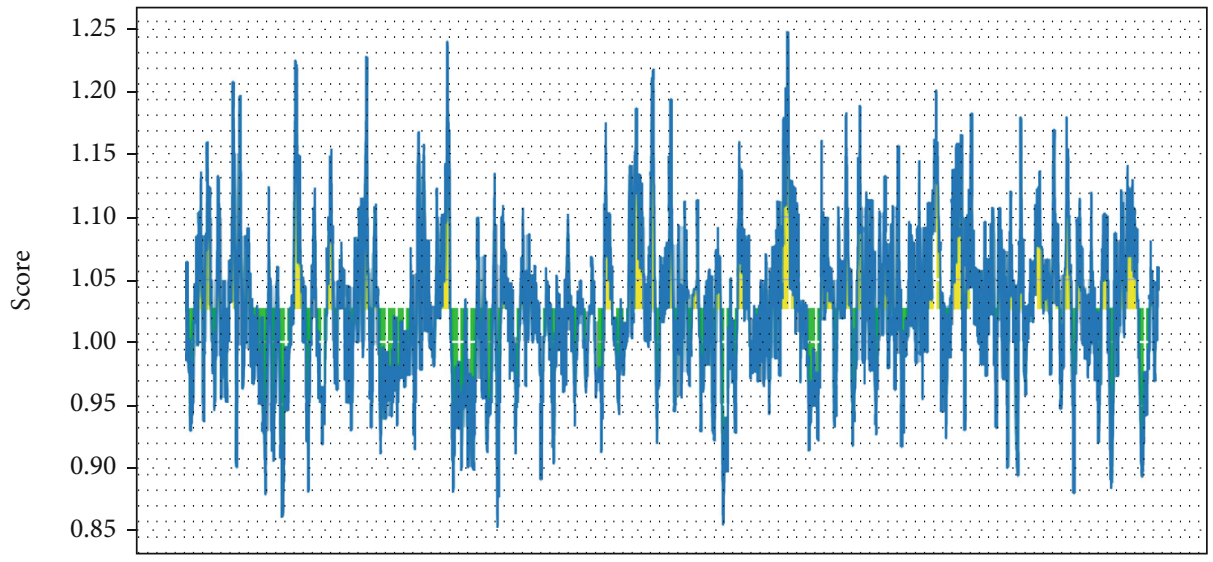

Position

(e)

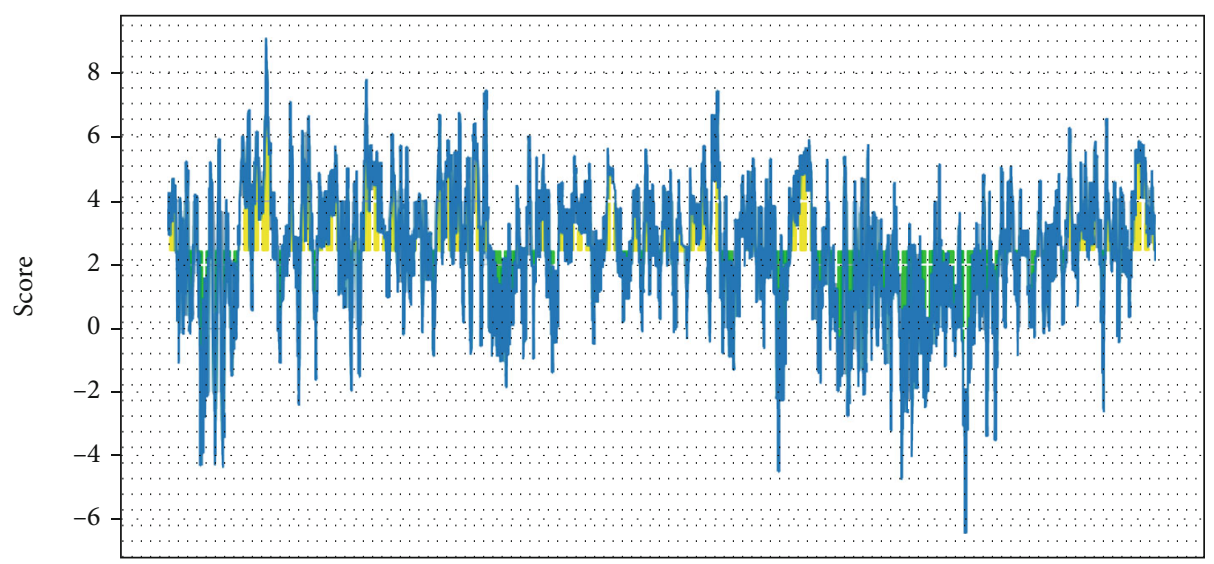

Position

(f)

FIGURE 6: Propensity scale plots of CDPK7 protein. (a) Bepipred linear; (b) beta-turn; (c) surface accessibility; (d) flexibility; (e) antigenicity; (f) hydrophilicity. $x$-axis and $y$-axis represent position and score, respectively. The horizontal line indicates the threshold or the average score. Yellow colors (above the threshold) indicate favorable regions related to the properties of interest. Green color (below the threshold) indicates the unfavorable regions related to the properties of interest. 
TABLE 4: Conformational B-cell epitopes of CDPK7 protein predicted by the ElliPro server.

\begin{tabular}{lcc}
\hline Residues & $\begin{array}{c}\text { Number of } \\
\text { residues }\end{array}$ & Score \\
\hline
\end{tabular}

A:V1431, A:L1432, A:Y1433, A:K1434, A:K1435, A:G1436, A:K1437, A:H1438, A:L1439, A:H1440, A:Q1441, A:W1442, A:Q1443, A:A1444, A:R1445, A:Y1456, A:Y1457, A:R1458, A:R1459, A:K1460, A:G1461, A:D1462, A:A1463, A:K1464, A:P1465, A:R1466, A:G1467, A:F1468, A:E1477, A:L1478, A:L1479, A:S1480, A:E1481, A:Q1482, A:V1483, A:G1484, A:G1485, A:R1486, A:Q1487, A:Y1488, A:G1489, A:L1504, A:L1505, A:F1506, A:A1507, A:N1508, A:S1509, A:A1510, A:K1511, A:Q1513, A:R1514

A:V1493, A:H1494, A:P1495, A:K1496, A:G1497, A:E1498, A:T1499, A:V1500, A:S1501, A:K1502, A:R1503

A:A1528, A:L1529, A:E1530, A:Q1531, A:L1532, A:Y1533, A:Q1534, A:V1535, A:G1536, A:E1537, A:Q1538, A:H1541, A:I1546, A:Y1548, A:K1549, A:G1550, A:I1551, A:H1552, A:R1553, A:A1554, A:T1555, A:N1556, A:E1557, A:L1558, A:L1611, A:V1612, A:R1613, A:G1614, A:Q1623, A:N1624, A:H1625, A:L1627, A:P1628, A:E1629, A:L1630, A:H1631, A:N1633, A:R1634, A:T1664, A:D1665, A:R1666, A:T1667, A:P1668, A:N1669, A:A1670, A:V1699, A:A1700, A:P1701, A:L1704, A:T1705, A:M1706, A:L1726, A:R1727, A:G1728, A:R1729, A:L1730, A:P1731, A:F1732, A:P1733, A:I1734, A:N1735, A:Q1736, A:A1737, A:F1738, A:G1739, A:P1741, A:S1742, A:F1743, A:Y1744, A:E1745, A:N1746, A:T1747, A:P1748, A:V1749, A:S1750, A:F1751, A:D1752, A:G1753, A:A1754, A:V1755, A:W1756, A:E1758, A:V1759, A:S1760, A:S1761, A:S1762, A:A1763, A:K1764, A:D1765, A:V1768, A:R1769, A:L1771, A:Q1772, A:P1773, A:N1774, A:P1775, A:R1776, A:R1777, A:R1778
51

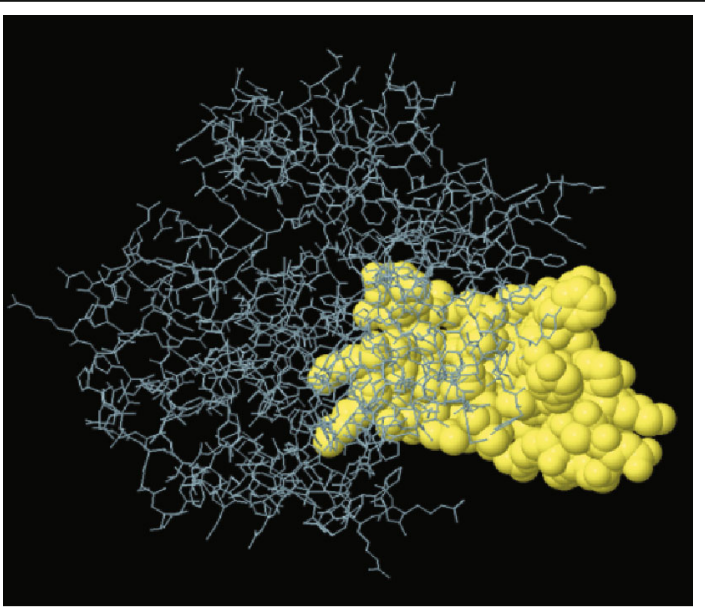

11

0.755

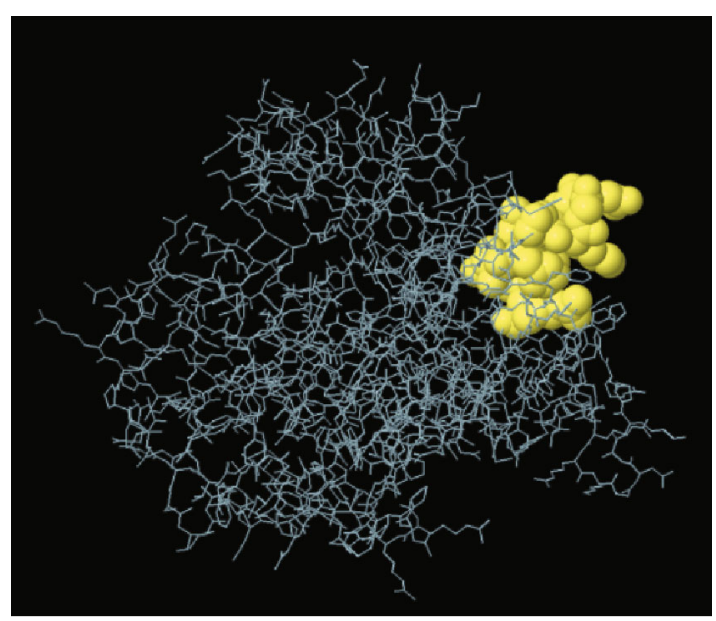

99

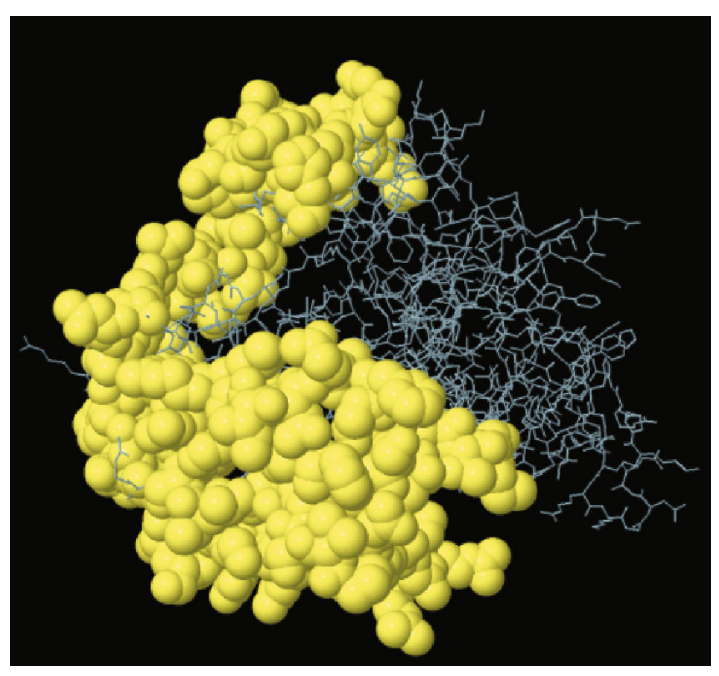


TABLE 4: Continued.

\begin{tabular}{lcc}
\hline Residues & $\begin{array}{c}\text { Number of } \\
\text { residues }\end{array}$ & Score \\
\hline
\end{tabular}

A:F1544, A:D1565, A:K1566, A:G1567, A:K1568, A:I1569, A:N1570, A:G1571, A:H1572, A:E1603, A:T1604, A:Y1606

A:C1683, A:A1684, A:P1685, A:N1686, A:E1687, A:V1688, A:L1689, A:Q1691, A:P1692, A:C1693
12

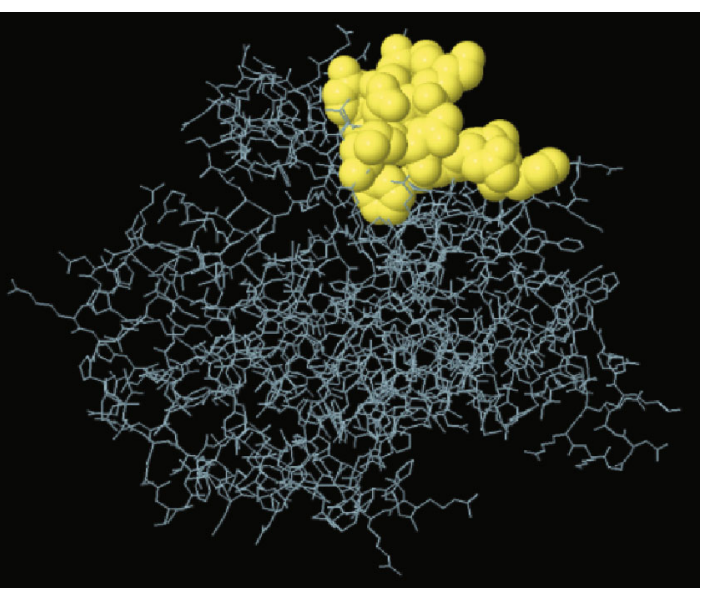

10

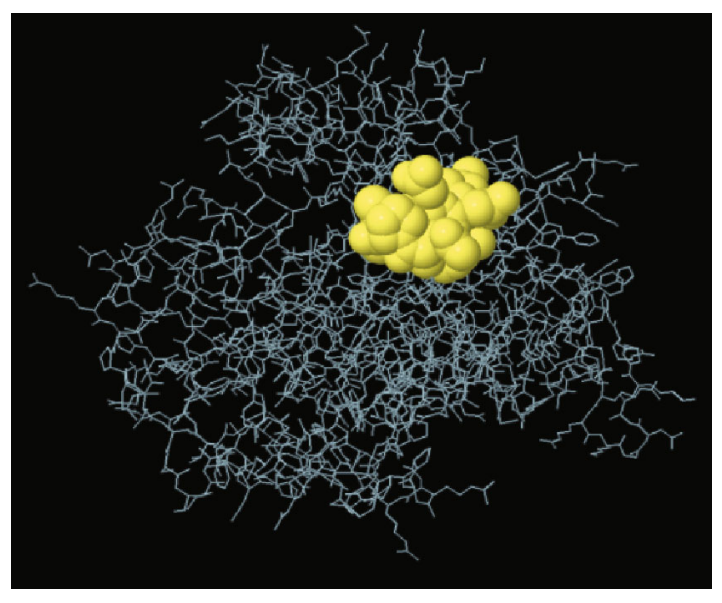

amino acid sequence of CDPK7 comprises 2133 residues with an average MW of $219085.79 \mathrm{D}$, which characterizes a suitable antigenic nature (the peptides with MW more than $10 \mathrm{kDa}$ are considered as good immunogens) [63]. According to the Expasy ProtParam server, GRAVY and the aliphatic index of the CDPK7 were achieved at -0.331 and 68.78, respectively. In summary, the great value of aliphatic index means that the peptide has more stability in a broad range of various temperatures. Moreover, the low/negative value of the GRAVY factor signifies the better interaction of peptide with the molecules of water. It is efficient to identify that PTMs have a fundamental role in cell stability [64]. The acquired outcomes show that CDPK7 comprises 299 potential PTM sites (269 phosphorylation and 30 acylation positions), representing that these positions may organize protein activity.

To predict the secondary structure of CDPK7, the GOR4 tool was recruited. The results of secondary structure of CDPK7 verified and included 502 (out of 2133) alpha-helix, 320 extended strands, and 1311 random coils. It is known that the key role of the proteins is related to their threedimensional structure. As such, to comprehend the influ- ences between both structures and functions, assessment of $3 \mathrm{D}$ structure is the key aim of expecting a protein's nature [65].

Humoral and cellular immunity are strongly stimulated in $T$. gondii infection $[66,67]$, in such a way that the establishment of IgG antibodies avoids the protozoan from attachment to the receptors of host cell [67]. Interferon- $\gamma$ (IFN- $\gamma$ ), $\mathrm{CD}_{4}{ }^{+}$, and $\mathrm{CD}_{8}{ }^{+} \mathrm{T}$ cells as the main members of T cells play a dynamic role in constraining acute and chronic infection. These major cytokines prevent the reactivation of bradyzoites in the host tissue cyst [66]. Epitope prediction has critical value to evaluate the specificity of antigen. Furthermore, epitope evaluation may reveal the pathogenesis and immune process of the pathogen in design vaccine researches $[65,68]$. The strength of using in silico is the detection of the component epitopes that are critical for the interaction of antibodies and antigens. Several linear B-cell epitopes were predicted by the ABCpred server, among which those epitopes above 0.9 score were of great significance to be included in multiepitope vaccine constructs. Moreover, we applied the IEDB online server to evaluate the $\mathrm{IC}_{50}$ values of peptides that link to the MHC class I/II molecules for CDPK7. According to the 
TABLE 5: $\mathrm{IC}_{50}$ values for CDPK7 binding to MHC class I molecules obtained using the IEDB ${ }^{\mathrm{a}}$.

\begin{tabular}{|c|c|c|c|c|}
\hline MHC-II allele ${ }^{\mathrm{b}}$ & $\begin{array}{l}\text { Start-stop }^{c} \\
\text { CDPK7 }\end{array}$ & Peptide sequence & $\begin{array}{l}\text { Percentile rank }^{\mathrm{d}} \\
\text { CDPK7 }\end{array}$ & Antigenicity \\
\hline \multirow{3}{*}{$\mathrm{H} 2-\mathrm{Db}$} & $1882-1891$ & SSPSSLPTPI & 0.15 & 0.4079 \\
\hline & $143-152$ & SNLPNLDRYM & 0.21 & -0.4284 \\
\hline & $1637-1646$ & SQLLSTVYYL* & 0.24 & 0.9146 \\
\hline \multirow{3}{*}{$\mathrm{H} 2-\mathrm{Dd}$} & $647-656$ & KGPTKSAMLL* & 0.18 & 0.9201 \\
\hline & $1612-1621$ & VRGGELFDLI & 0.28 & 0.1770 \\
\hline & $1483-1492$ & VGGRQYGFAI & 0.64 & 0.1561 \\
\hline \multirow{3}{*}{$\mathrm{H} 2-\mathrm{Kb}$} & $1721-1730$ & IMYLLLRGRL* & 0.55 & 1.4751 \\
\hline & $798-807$ & SSASFSSRGM* & 1.0 & 1.3623 \\
\hline & $1643-1652$ & VYYLHKCGIV & 1.2 & 0.1373 \\
\hline \multirow{3}{*}{$\mathrm{H} 2-\mathrm{Kd}$} & $1474-1483$ & CYVELLSEQV* & 0.79 & 0.5079 \\
\hline & $1445-1454$ & RYYVLVDNML* & 1.15 & 0.9079 \\
\hline & $822-831$ & GYSASGGMIV* & 1.3 & 0.9579 \\
\hline \multirow{3}{*}{$\mathrm{H} 2-\mathrm{Kk}$} & $1555-1564$ & TNELYAIKVI & 0.12 & 0.2695 \\
\hline & $1084-1093$ & SEGPATTPSI & 0.75 & 0.2366 \\
\hline & $694-703$ & DVEGIVDKII & 1.5 & 0.1511 \\
\hline \multirow{3}{*}{$\mathrm{H} 2-\mathrm{Ld}$} & $1589-1598$ & HPNVIYMKEL & 3.8 & 0.2773 \\
\hline & $1729-1738$ & RLPFPINQAF & 4.2 & 0.3317 \\
\hline & $279-288$ & YEPHPLLARL & 4.6 & 0.1027 \\
\hline
\end{tabular}

${ }^{\mathrm{a}}$ The immune epitope database (http://tools.iedb.org/mhci/). ${ }^{\mathrm{b}} \mathrm{H} 2-\mathrm{Db}, \mathrm{H} 2-\mathrm{Dd}, \mathrm{H} 2-\mathrm{Kb}, \mathrm{H} 2-\mathrm{Kd}, \mathrm{H} 2-\mathrm{Kk}$, and $\mathrm{H} 2-\mathrm{Ld}$ alleles are mouse MHC class I molecules. ${ }^{c}$ Ten amino acids for analysis were used each time. ${ }^{\mathrm{d}}$ Low percentile rank $=$ high level binding; high percentile rank $=$ low level binding; $\mathrm{IC}_{50}$ values $=$ percentile rank. $*$ indicates potential antigenic epitopes (threshold $=0.5$ ).

TABLE 6: IC $_{50}$ values for CDPK7 binding to MHC class II molecules obtained using the IEDB ${ }^{\mathrm{a}}$.

\begin{tabular}{|c|c|c|c|c|}
\hline MHC-II allele ${ }^{b}$ & $\begin{array}{l}\text { Start-stop }^{c} \\
\text { CDPK7 }\end{array}$ & Peptide sequence & $\begin{array}{l}\text { Percentile rank } \\
\text { CDPK7 }\end{array}$ & Antigenicity \\
\hline \multirow{3}{*}{$\mathrm{H} 2-\mathrm{IAb}$} & $1109-1123$ & AAGAAAAAATAAAAA* & 0.07 & 0.8045 \\
\hline & $1108-1122$ & AAAGAAAAAATAAAA* & 0.08 & 0.8354 \\
\hline & $1110-1124$ & AGAAAAAATAAAAAF* & 0.08 & 0.7176 \\
\hline \multirow{3}{*}{ H2-IAd } & $1035-1049$ & SETQPAMASVASGSS* & 0.13 & 0.6766 \\
\hline & $1034-1048$ & GSETQPAMASVASGS* & 0.15 & 0.7059 \\
\hline & $1036-1050$ & ETQPAMASVASGSSP* & 0.25 & 0.6536 \\
\hline \multirow{3}{*}{ H2-IEd } & $1451-1465$ & DNMLYYYRRKGDAKP* & 0.14 & 0.6972 \\
\hline & $1452-1466$ & NMLYYYRRKGDAKPR* & 0.14 & 0.8298 \\
\hline & $1450-1464$ & VDNMLYYYRRKGDAK* & 0.19 & 0.6159 \\
\hline
\end{tabular}

${ }^{a}$ The immune epitope database (http://tools.immuneepitope.org/mhcii). ${ }^{b} \mathrm{H} 2$-IAb, H2-IAd, and H2-IEd alleles are mouse MHC class II molecules. ${ }^{c}$ Fifteen amino acids for analysis were used each time. ${ }^{\mathrm{d}}$ Low percentile rank $=$ high level binding; high percentile rank $=$ low level binding; $\mathrm{IC}_{50}$ values $=$ percentile rank. $*$ indicates potential antigenic epitopes (threshold $=0.5$ ).

obtained results from IEDB, the T-cell epitopes on CDPK7 have the capability to bind intensely to MHC class I and class II molecules. It is important to note that the lower $\mathrm{IC}_{50}$ values show the higher-level of affinity, which show an appropriate T-cell epitope.

Other the main stage, CTLpred is a special approach used to predict CTL epitopes, which is important in vaccine- related studies. This tool relies on elegant machine learning methods, such as ANN and SVM. We recognized the CTL epitopes using the CTLpred online database to select the top CDPK7 epitopes. The CTLpred server utilizes consensus and combined estimates, in line with these two methods [56]. Evaluation of antigenicity and allergenicity showed that CDPK7 protein has immunogenic and nonallergenic nature. 
TABLe 7: Predicted CDPK7 epitopes by CTLpred ${ }^{\mathrm{a}}$.

\begin{tabular}{|c|c|c|c|c|}
\hline Peptide rank & Start position ${ }^{\mathrm{b}}$ & Sequence & Score $\left(\right.$ ANN/SVM) ${ }^{c}$ & Antigenicity \\
\hline 1 & 280 & EPHPLLARL & $0.83 / 1.3591088$ & 0.0131 \\
\hline 2 & 1716 & WSIGVIMYL & $0.96 / 1.1120848$ & 0.1711 \\
\hline 3 & 1398 & GSSRVFTRC & $0.94 / 1.0685326$ & -0.7197 \\
\hline 4 & 1187 & ARDDDVYER & $0.65 / 1.3441588$ & 0.3493 \\
\hline 5 & 715 & SFPEFKTWL ${ }^{*}$ & $0.98 / 0.95345497$ & 1.0485 \\
\hline 6 & 1763 & AKDLIVRML* & $0.98 / 0.89030833$ & 0.8096 \\
\hline 7 & 724 & ERNEGILSM $^{*}$ & $0.65 / 1.0757075$ & 0.5393 \\
\hline 8 & 470 & ASRFTSAIK* & $0.80 / 0.85963689$ & 1.0303 \\
\hline 9 & 1573 & ERELLRSEM ${ }^{*}$ & $0.51 / 1.0720792$ & 0.9337 \\
\hline 10 & 1188 & RDDDVYERI & $0.85 / 0.73017891$ & 0.0942 \\
\hline 11 & 1666 & RTPNATIKL & $0.99 / 0.58481613$ & 0.2323 \\
\hline 12 & 32 & KECLKQYLK* & $0.99 / 0.58376856$ & 1.2628 \\
\hline 13 & 1411 & WELSKCAEM & $0.19 / 1.3750392$ & 0.3168 \\
\hline 14 & 1749 & VSFDGAVWR* & $0.96 / 0.59370426$ & 1.2284 \\
\hline 15 & 743 & GLQGNALYR* & $0.99 / 0.54484483$ & 1.4369 \\
\hline
\end{tabular}

${ }^{a}$ CTLpred, available online at http://www.imtech.res.in/raghava/ctlpred/index.html. ${ }^{b}$ Nine amino acids for analysis were used. ${ }^{c}$ The default artificial neural network (ANN) and support vector machine (SVM) cut-off scores were set 0.51 and 0.36 , respectively. * indicates potential antigenic epitopes (threshold $=0.5$ ).

\section{Conclusion}

Well antigenicity, hydrophilicity, surface accessibility, and flexibility indexes were detected for CDPK7. Hence, we recommend that a suitable vaccine should be designed and verified both in silico and in vivo by the potential B- and T-cell epitopes predicted in this study.

\section{Abbreviations}

3D: $\quad$ Three-dimensional

ACC: $\quad$ Auto cross covariance

ANN: Artificial neural network

CD: $\quad$ Cluster of differentiation

CDPK: Calcium-dependent protein kinase

CTL: $\quad$ Cytotoxic T-lymphocyte

GOR: Garnier-Osguthorpe-Robson

GRAVY: Grand average of hydropathicity

$\mathrm{IC}_{50}$ : Half-maximal inhibitory concentration

IEDB: Immune epitope database

IFN- $\gamma$ : Interferon- $\gamma$

MHC: $\quad$ Major histocompatibility complex

MW: Molecular weight

PDB: $\quad$ Protein data bank

pI: $\quad$ Isoelectric point

PTM: $\quad$ Post-translational modification

SVM: $\quad$ Support vector machine

T. gondii: Toxoplasma gondii.

\section{Data Availability}

The datasets used and/or analysed during the current study are available from the corresponding author on reasonable request.

\section{Ethical Approval}

This study received the approval from the Behbahan Faculty of Medical Sciences Ethical Committee (IR.BHN.REC.1399.034).

\section{Disclosure}

The funders of this study had no role in the study design, analysis and interpretation of data, writing of the final paper, and the decision to submit the manuscript for publication. The corresponding author had access to the data in the study and had final responsibility for the decision to submit for publication.

\section{Conflicts of Interest}

The authors declare that there is no conflict of interest.

\section{Acknowledgments}

This study was financially supported by the Behbahan Faculty of Medical Sciences, Behbahan, Iran (Grant No. 99013).

\section{References}

[1] J. P. Dubey, "The history ofToxoplasma gondiiâ The first 100 years," The Journal of Eukaryotic Microbiology, vol. 55, no. 6, pp. 467-475, 2008.

[2] M. Foroutan, Y. Fakhri, S. M. Riahi et al., "The global seroprevalence of Toxoplasma gondii in pigs: A systematic review and meta-analysis," Veterinary Parasitology, vol. 269, pp. 42-52, 2019.

[3] J. Dubey, "History of the discovery of the life cycle of Toxoplasma gondii," International Journal for Parasitology, vol. 39, no. 8, pp. 877-882, 2009. 
[4] K. Shapiro, L. Bahia-Oliveira, B. Dixon et al., "Environmental transmission of Toxoplasma gondii: Oocysts in water, soil and food," Food and Waterborne Parasitology, vol. 15, article e00049, 2019.

[5] M. Foroutan-Rad, H. Majidiani, S. Dalvand et al., "Toxoplasmosis in blood donors: a systematic review and meta-analysis," Transfusion Medicine Reviews, vol. 30, no. 3, pp. 116-122, 2016.

[6] A. Rostami, S. M. Riahi, D. G. Contopoulos-Ioannidis et al., "Acute Toxoplasma infection in pregnant women worldwide: a systematic review and meta-analysis," PLoS Neglected Tropical Diseases, vol. 13, no. 10, article e0007807, 2019.

[7] B. Maleki, N. Ahmadi, M. Olfatifar et al., "Toxoplasma oocysts in the soil of public places worldwide: a systematic review and meta-analysis," Transactions of the Royal Society of Tropical Medicine and Hygiene, vol. 115, no. 5, pp. 471-481, 2021.

[8] A. Rostami, S. M. Riahi, H. R. Gamble et al., "Global prevalence of latent toxoplasmosis in pregnant women: a systematic review and meta-analysis," Clinical Microbiology and Infection, vol. 26, no. 6, pp. 673-683, 2020.

[9] Z. D. Wang, H. H. Liu, Z. X. Ma et al., "Toxoplasma gondii infection in immunocompromised patients: a systematic review and meta-analysis," Frontiers in Microbiology, vol. 8, p. 389, 2017.

[10] S. Soltani, M. S. Kahvaz, S. Soltani, F. Maghsoudi, and M. Foroutan, "Seroprevalence and associated risk factors of Toxoplasma gondii infection in patients undergoing hemodialysis and healthy group," BMC Research Notes, vol. 13, no. 1, p. 551, 2020.

[11] H. Furrer, M. Opravil, E. Bernasconi, A. Telenti, and M. Egger, "Stopping primary prophylaxis in HIV-1-infected patients at high risk of toxoplasma encephalitis," The Lancet, vol. 355, no. 9222, pp. 2217-2218, 2000.

[12] S. Fallahi, A. Rostami, M. Nourollahpour Shiadeh, H. Behniafar, and S. Paktinat, "An updated literature review on maternal-fetal and reproductive disorders of Toxoplasma gondii infection," Journal of Gynecology Obstetrics and Human Reproduction, vol. 47, no. 3, pp. 133-140, 2018.

[13] R. Silva, H. Langoni, and J. Megid, "Adaptive and genetic evolution of Toxoplasma gondii: a host-parasite interaction," Revista da Sociedade Brasileira de Medicina Tropical, vol. 50, no. 4, pp. 580-581, 2017.

[14] L. Galal, D. Ajzenberg, A. Hamidović, M. F. Durieux, M. L. Dardé, and A. Mercier, "Toxoplasma and Africa: One Parasite, Two Opposite Population Structures," Trends in Parasitology, vol. 34, no. 2, pp. 140-154, 2018.

[15] M. Antczak, K. Dzitko, and H. Długońska, "Human toxoplasmosis-Searching for novel chemotherapeutics," Biomedicine \& Pharmacotherapy, vol. 82, pp. 677-684, 2016.

[16] S. Rajapakse, M. Chrishan Shivanthan, N. Samaranayake, C. Rodrigo, and S. Deepika Fernando, "Antibiotics for human toxoplasmosis: a systematic review of randomized trials," Pathogens and Global Health, vol. 107, no. 4, pp. 162-169, 2013.

[17] P. Valentini, M. Annunziata, D. F. Angelone et al., "Role of spiramycin/cotrimoxazole association in the mother-to-child transmission of toxoplasmosis infection in pregnancy," European Journal of Clinical Microbiology \& Infectious Diseases, vol. 28, no. 3, pp. 297-300, 2009.

[18] N.-Z. Zhang, M. Wang, Y. Xu, E. Petersen, and X. Q. Zhu, "Recent advances in developing vaccines against Toxoplasma gondii: an update," Expert Review of Vaccines, vol. 14, no. 12, pp. 1609-1621, 2015.

[19] M. Foroutan, L. Zaki, S. Tavakoli, S. Soltani, A. Taghipour, and F. Ghaffarifar, "Rhomboid antigens are promising targets in the vaccine development against Toxoplasma gondii," EXCLI Journal, vol. 18, pp. 259-272, 2019.

[20] M. Foroutan, F. Ghaffarifar, Z. Sharifi, A. Dalimi, and O. Jorjani, "Rhoptry antigens asToxoplasma gondiivaccine target," Clinical and Experimental Vaccine Research, vol. 8, no. 1, pp. 4-26, 2019.

[21] M. Foroutan, L. Zaki, and F. Ghaffarifar, "Recent progress in microneme-based vaccines development againstToxoplasma gondii," Clinical and Experimental Vaccine Research, vol. 7, no. 2, pp. 93-103, 2018.

[22] A. Asghari, S. Shamsinia, H. Nourmohammadi et al., "Development of a chimeric vaccine candidate based on Toxoplasma gondii major surface antigen 1 and apicoplast proteins using comprehensive immunoinformatics approaches," European Journal of Pharmaceutical Sciences, vol. 162, p. 105837, 2021.

[23] M. C. Nosrati, E. Ghasemi, M. Shams et al., "Toxoplasma gondii ROP38 protein: Bioinformatics analysis for vaccine design improvement against toxoplasmosis," Microbial Pathogenesis, vol. 149, p. 104488, 2020.

[24] H. Can, S. Erkunt Alak, A. E. Köseoğlu, M. Döşkaya, and C. Ün, "Do Toxoplasma gondii apicoplast proteins have antigenic potential? An in silico study," Computational Biology and Chemistry, vol. 84, p. 107158, 2020.

[25] A. D. Ghaffari, A. Dalimi, F. Ghaffarifar, M. Pirestani, and H. Majidiani, "Immunoinformatic analysis of immunogenic B- and T-cell epitopes of MIC4 protein to designing a vaccine candidate against Toxoplasma gondii through an in-silico approach," Clinical and Experimental Vaccine Research, vol. 10, no. 1, pp. 59-77, 2021.

[26] H. Majidiani, A. Dalimi, F. Ghaffarifar, M. Pirestani, and A. D. Ghaffari, "Computational probing of Toxoplasma gondii major surface antigen 1 (SAG1) for enhanced vaccine design against toxoplasmosis," Microbial Pathogenesis, vol. 147, p. 104386, 2020.

[27] A. D. Ghaffari, A. Dalimi, F. Ghaffarifar, and M. Pirestani, "Structural predication and antigenic analysis of ROP16 protein utilizing immunoinformatics methods in order to identification of a vaccine against Toxoplasma gondii: An in silico approach," Microbial Pathogenesis, vol. 142, p. 104079, 2020.

[28] A. D. Ghaffari, A. Dalimi, F. Ghaffarifar, and M. Pirestani, "Antigenic properties of dense granule antigen 12 protein using bioinformatics tools in order to improve vaccine design against Toxoplasma gondii," Clinical and Experimental Vaccine Research, vol. 9, no. 2, pp. 81-96, 2020.

[29] M. Foroutan, A. D. Ghaffari, S. Soltani, H. Majidiani, A. Taghipour, and M. Sabaghan, "Bioinformatics analysis of calcium-dependent protein kinase 4 (CDPK4) as Toxoplasma gondii vaccine target," BMC Research Notes, vol. 14, no. 1, p. 50, 2021.

[30] M. Khodadadi, F. Ghaffarifar, A. Dalimi, and E. Ahmadpour, "Immunogenicity of in-silico designed multi-epitope DNA vaccine encoding SAG1, SAG3 and SAG5 of Toxoplasma gondii adjuvanted with CpG-ODN against acute toxoplasmosis in BALB/c mice," Acta Tropica, vol. 216, p. 105836, 2021.

[31] M. Foroutan, F. Ghaffarifar, Z. Sharifi, and A. Dalimi, "Vaccination with a novel multi-epitope ROP8 DNA vaccine against acute Toxoplasma gondii infection induces strong $\mathrm{B}$ and $\mathrm{T}$ cell 
responses in mice," Comparative Immunology, Microbiology and Infectious Diseases, vol. 69, p. 101413, 2020.

[32] M. Foroutan, F. Ghaffarifar, Z. Sharifi, A. Dalimi, and M. Pirestani, "Bioinformatics analysis of ROP8 protein to improve vaccine design against Toxoplasma gondii," Infection, Genetics and Evolution, vol. 62, pp. 193-204, 2018.

[33] M. Tzen, R. Benarous, J. Dupouy-Camet, and M. P. Roisin, “A novel Toxoplasma gondii calcium-dependent protein kinase," Parasite, vol. 14, no. 2, pp. 141-147, 2007.

[34] M. Foroutan and F. Ghaffarifar, "Calcium-dependent protein kinases are potential targets for Toxoplasma gondii vaccine," Clinical and experimental vaccine research, vol. 7, no. 1, pp. 24-36, 2018.

[35] J. Morlon-Guyot, L. Berry, C. T. Chen, M. J. Gubbels, M. Lebrun, and W. Daher, "The Toxoplasma gondii calciumdependent protein kinase 7 is involved in early steps of parasite division and is crucial for parasite survival," Cellular Microbiology, vol. 16, no. 1, pp. 95-114, 2014.

[36] D. R. Flower, "Computer-aided vaccine design," Human Vaccines \& Immunotherapeutics, vol. 10, no. 1, pp. 241243, 2014.

[37] E. Gasteiger, C. Hoogland, A. Gattiker et al., "Protein Identification and Analysis Tools on the ExPASy Server," in The proteomics protocols handbook, pp. 571-607, Springer, 2005.

[38] J. Zhou, L. Wang, A. Zhou et al., "Bioinformatics analysis and expression of a novel protein ROP48 in Toxoplasma gondii," Acta Parasitologica, vol. 61, no. 2, pp. 319-328, 2016.

[39] H. Majidiani, S. Soltani, A. D. Ghaffari, M. Sabaghan, A. Taghipour, and M. Foroutan, "In-depth computational analysis of calcium-dependent protein kinase 3 of Toxoplasma gondii provides promising targets for vaccination," Clin Exp Vaccine Res, vol. 9, no. 2, pp. 146-158, 2020.

[40] J. Garnier, J.-F. Gibrat, and B. Robson, "GOR method for predicting protein secondary structure from amino acid sequence," in Methods Enzymol, pp. 540-553, Elsevier, 1996.

[41] N. Guex, M. C. Peitsch, and T. Schwede, "Automated comparative protein structure modeling with SWISS-MODEL and Swiss-PdbViewer: a historical perspective," Electrophoresis, vol. 30, no. S1, pp. S162-S173, 2009.

[42] H. Park and C. Seok, "Refinement of unreliable local regions in template-based protein models," Proteins: Structure, Function, and Bioinformatics, vol. 80, pp. 1974-1986, 2012.

[43] M. Bertoni, F. Kiefer, M. Biasini, L. Bordoli, and T. Schwede, "Modeling protein quaternary structure of homo- and hetero-oligomers beyond binary interactions by homology," Scientific Reports, vol. 7, no. 1, p. 10480, 2017.

[44] M. Wiederstein and M. J. Sippl, "ProSA-web: interactive web service for the recognition of errors in three-dimensional structures of proteins," Nucleic Acids Research, vol. 35, no. Web Server, pp. W407-W410, 2007.

[45] S. Saha and G. P. S. Raghava, "BcePred: prediction of continuous B-cell epitopes in antigenic sequences using physicochemical properties," in International Conference on Artificial Immune Systems, pp. 197-204, Springer, 2004.

[46] S. Saha and G. P. S. Raghava, "Prediction of continuous B-cell epitopes in an antigen using recurrent neural network," Proteins: Structure, Function, and Bioinformatics, vol. 65, no. 1, pp. 40-48, 2006.

[47] J. M. R. Parker, D. Guo, and R. S. Hodges, "New hydrophilicity scale derived from high-performance liquid chromatography peptide retention data: correlation of predicted surface resi- dues with antigenicity and X-ray-derived accessible sites," Biochemistry, vol. 25, no. 19, pp. 5425-5432, 1986.

[48] J. E. P. Larsen, O. Lund, and M. Nielsen, "Improved method for predicting linear B-cell epitopes," Immunome Research, vol. 2, no. 1, p. 2, 2006.

[49] A. Kolaskar and P. C. Tongaonkar, "A semi-empirical method for prediction of antigenic determinants on protein antigens," FEBS Letters, vol. 276, no. 1-2, pp. 172-174, 1990.

[50] E. A. Emini, J. V. Hughes, D. Perlow, and J. Boger, "Induction of hepatitis A virus-neutralizing antibody by a virus-specific synthetic peptide," Journal of Virology, vol. 55, no. 3, pp. 836-839, 1985.

[51] P. Y. Chou and G. D. Fasman, "Prediction of the secondary structure of proteins from their amino acid sequence," Advances in Enzymology and Related Areas of Molecular Biology, vol. 47, pp. 45-148, 1978.

[52] P. Karplus and G. E. Schulz, "Prediction of chain flexibility in proteins," Naturwissenschaften, vol. 72, no. 4, pp. 212-213, 1985.

[53] J. Ponomarenko, H.-H. Bui, W. Li et al., "ElliPro: a new structure-based tool for the prediction of antibody epitopes," BMC Bioinformatics, vol. 9, no. 1, p. 514, 2008.

[54] M. Andreatta and M. Nielsen, "Gapped sequence alignment using artificial neural networks: application to the MHC class I system," Bioinformatics, vol. 32, no. 4, pp. 511-517, 2016.

[55] P. Wang, J. Sidney, C. Dow, B. Mothé, A. Sette, and B. Peters, "A systematic assessment of MHC class II peptide binding predictions and evaluation of a consensus approach," PLoS Computational Biology, vol. 4, no. 4, article e1000048, 2008.

[56] M. Bhasin and G. P. S. Raghava, "Prediction of CTL epitopes using QM, SVM and ANN techniques," Vaccine, vol. 22, no. 23-24, pp. 3195-3204, 2004.

[57] I. A. Doytchinova and D. R. Flower, "VaxiJen: a server for prediction of protective antigens, tumour antigens and subunit vaccines," BMC Bioinformatics, vol. 8, no. 1, p. 4, 2007.

[58] I. Dimitrov, L. Naneva, I. Doytchinova, and I. Bangov, "AllergenFP: allergenicity prediction by descriptor fingerprints," Bioinformatics, vol. 30, no. 6, pp. 846-851, 2014.

[59] I. Dimitrov, D. R. Flower, and I. Doytchinova, "AllerTOP-a server for in silico prediction of allergens," in BMC bioinformatics, p. S4, BioMed Central, 2013.

[60] S. Stelzer, W. Basso, J. Benavides Silván et al., “Toxoplasma gondii infection and toxoplasmosis in farm animals: Risk factors and economic impact," Food and Waterborne Parasitology, vol. 15, article e00037, 2019.

[61] F. Rezaei, S. Sarvi, M. Sharif et al., "A systematic review of Toxoplasma gondii antigens to find the best vaccine candidates for immunization," Microbial Pathogenesis, vol. 126, pp. 172184, 2019.

[62] S. Parvizpour, M. M. Pourseif, J. Razmara, M. A. Rafi, and Y. Omidi, "Epitope-based vaccine design: a comprehensive overview of bioinformatics approaches," Drug Discovery Today, vol. 25, no. 6, pp. 1034-1042, 2020.

[63] J. Berzofsky and I. Berkower, "Antigen-antibody interaction," in Fundamental immunology, pp. 595-644, Raven Press, New York, 1984.

[64] T.-Y. Lee, J. B.-K. Hsu, W.-C. Chang, T. Y. Wang, P. C. Hsu, and H. D. Huang, "A comprehensive resource for integrating and displaying protein post-translational modifications," BMC Research Notes, vol. 2, no. 1, p. 111, 2009. 
[65] Y. Wang, G. Wang, J. Cai, and H. Yin, "Review on the identification and role of Toxoplasma gondii antigenic epitopes," Parasitology Research, vol. 115, no. 2, pp. 459-468, 2016.

[66] I. El-Kady, "T-cell immunity in human chronic toxoplasmosis," Journal of the Egyptian Society of Parasitology, vol. 41, no. 1, pp. 17-28, 2011.

[67] P. C. Sayles, G. W. Gibson, and L. L. Johnson, "B cells are essential for vaccination-induced resistance to virulent Toxoplasma gondii," Infection and Immunity, vol. 68, no. 3, pp. 1026-1033, 2000.

[68] D. Xu and Y. Zhang, "Improving the physical realism and structural accuracy of protein models by a two-step atomiclevel energy minimization," Biophysical Journal, vol. 101, no. 10, pp. 2525-2534, 2011. 